\title{
Pioneer Growth Cone Morphologies Reveal Proximal Increases in Substrate Affinity Within Leg Segments of Grasshopper Embryos
}

\author{
Michael Caudy` and David Bentley $\dagger$ \\ *Biophysics Group and †Department of Zoology, University of California, Berkeley, California 94720
}

We have compared the morphologies of approximately 5000 antibody-labeled afferent pioneer growth cones fixed at various stages of growth along their characteristic path over the epithelium in the legs of grasshopper embryos, and have used growth cone morphology as an indicator of differences in the affinity of the epithelial substrate for pioneer growth cones in vivo. Growth cone morphologies differ markedly between different locations in limb buds, and also in the same location in limbs at different stages of differentiation. Growth cones characteristically extend branches and lamellae circumferentially along segment boundaries, and filopodia and lamellae are retained (or extended) longer there. Where they contact a relatively well-differentiated segment boundary, the growth cones also abruptly reorient circumferentially. In the proximal regions of limb segments, growth cones consistently have a high degree of branching and lamellae; previously formed axons also extend secondary branches and spread there as development progresses. Low incidence of these morphologies is observed at all stages in the distal regions of limb segments. Thus, neuronal morphologies correlate both spatially and temporally with the differentiation of limb segmentation.

These results suggest the following: (1) Detailed growth cone morphology is a reliable indicator of differences in extrinsic guidance cues. (2) The affinity of the epithelial substrate for afferent pioneer growth cones increases proximally within segments, with a peak at the segment boundary. (This affinity could be based on surface density of adhesion molecules or on nonadhesive molecules that actively regulate growth cone extension.) (3) Increasing epithelial affinity within segments appears to act as a proximal guidance cue for afferent pioneer growth cones. Pioneer growth cones are observed to navigate proximally in circumstances where proximally located guidepost cells differentiate too late to guide them.

During the course of development, the characteristic nerve branches seen in the adult are established by groups of axons that follow the same specific pathway. Many axons are guided over these long distances by fasciculating with previously formed axons. However, the initial axons must "pioneer" a pathway through an axonless environment.

Afferent pioneer axons establish the first neural pathways in grasshopper legs (Bate, 1976; Keshishian, 1980). Two extrinsic cues are known to guide these initial (Ti1) pioneer growth cones:

\footnotetext{
Received June 3, 1985; revised July 23, 1985; accepted July 26, 1985 .

We thank Alma Toroian-Raymond for light-microscope histology and technical assistance, Drs. Marty Shankland and Mark Cooper for helpful criticism of the manuscript, and Dr. Wolfram Kutsch for translating the German literature. Support was provided by Grants NIH NS09074-14 and NIH 2T32-GM07379. D.B. was also supported by an NIH Jacob Javits Award, and M.C. by an Einstein Fellowship for Developmental Neuroscience from the University of California.

Correspondence should be addressed to Michael Caudy, Department of Zoology, University of California, Berkeley, CA 94720.

Copyright (C) 1986 Society for Neuroscience $0270-6474 / 86 / 020364-16 \$ 02.00 / 0$
}

limb segment boundaries (Bentley and Caudy, 1983b) and guidepost cells. The dominant mechanism appears to be the guidepost cells, a set of nonadjacent, axonless cell bodies of immature neurons (Bentley and Caudy, 1983a, b; Bentley and Keshishian, 1982a, b; Ho and Goodman, 1982; Keshishian and Bentley, 1983a-c; Taghert et al., 1982). Ablation of the most proximal guidepost cell pair has been shown to result in lack of normal growth (Bentley and Caudy, 1983a, b).

Additional mechanisms apparently guide the Til growth cones proximally before they contact any of the above cues (Bentley and Caudy, 1983b). One possible external cue is an adhesion gradient on the epithelial substrate over which the pioneer growth cones navigate (Bentley and Caudy, 1983b; Berlot and Goodman, 1984). Nardi (1983) has presented evidence that a proximally increasing adhesion gradient on the epithelial substrate is a cue for afferent nerve fibers in moth wings.

In culture, growth cones have been shown to exhibit different morphologies on substrates known to be of different relative adhesivity (Letourneau, 1975a, 1979). Similar changes in morphology also accompany changes in the external concentration of NGF (Connolly et al., 1985), which appears to actively regulate neuronal adhesion or extension (Connolly et al., 1985; Schubert and Whitlock, 1977). If extrinsic guidance cues can determine pioneer growth cone morphology, then differences in growth cone morphology may reveal differences in extrinsic cues (Mason, 1985). Our analysis of pioneer growth cone morphology suggests that a proximally increasing pattern of epithelial affinity within leg segments contributes to growth cone guidance.

\section{Materials and Methods}

\section{Antibody labeling}

Animals were obtained from a colony of the grasshopper, Schistocerca americana, maintained at Berkeley. Embryos were dissected in saline (Bentley et al., 1979) and then immediately placed in saline containing $4 \%$ formaldehyde, $\mathrm{pH} 7.2$, for $1-7 \mathrm{~d}$. They were then rinsed in $0.1 \mathrm{M}$ PBS with $0.5 \%$ Triton (1 hr). (All subsequent steps prior to mounting had $0.5 \%$ Triton and were at room temperature.) The tissue was then incubated overnight in $0.02 \%$ rabbit anti-HRP serum (Cappel), which selectively labels insect neurons (Jan and Jan, 1982). This was followed by a $1 \mathrm{hr}$ rinse in PBS with 1\% BSA (Sigma) and incubation in $0.04 \%$ rhodamine-conjugated goat anti-rabbit serum (IgG fraction; U.S. Biochemical). Embryos were then rinsed in PBS with $1 \%$ BSA $(1 \mathrm{hr})$ and whole-mounted under coverglass with $40 \mu \mathrm{m}$ wire spacers in 1:9 PBS: glycerol, pH 7.2, with $10 \mathrm{mg} / \mathrm{ml}$ phenylenediamine (with or without 5 $\mathrm{mg} / \mathrm{ml}$ catechol) as an antioxidant. Slides were stored at $-20^{\circ} \mathrm{C}$ and viewed and photographed with a Zeiss epifluorescence microscope.

\section{Hoechst/antibody double-labeling}

Embryos were taken from secondary antibody as above and rinsed for $1 \mathrm{hr}$ in $0.1 \mathrm{~m} \mathrm{TBS}, \mathrm{pH} 7.3$, without Triton. They were then incubated overnight $\left(4^{\circ} \mathrm{C}\right)$ in TBS with $4 \%$ paraformaldehyde, $\mathrm{pH} 7.3$, containing $0.03 \mu \mathrm{g} / \mathrm{ml} \mathrm{Hoechst} 33258$ (Calbiochem), followed by a $1 \mathrm{hr}$ rinse in TBS, pH 9.0, and whole-mounted on slides with wire $40 \mu \mathrm{m}$ spacers in 
1:9 TBS : glycerol mountant, $\mathrm{pH} 9.0$, with antioxidants, as above. Slides were stored and viewed as above.

\section{Staging of pioneer neuron differentiation}

Stages are measured in percent of embryonic development; e.g., the $50 \%$ stage is halfway between fertilization and hatching (Bentley et al., 1979). The relative time of differentiation of an individual cell or morphological feature can vary by $\pm 1-2 \%$ relative to that of other cells or features in the leg. It is possible to identify accurately an "averaged stage" for a particular animal or leg by considering several morphological features, but within that average different variations can occur. Therefore, for convenience in describing pioneer axonogenesis, we define $1 \%$ stages in a particular leg by the usual location of the pioneer growth cones in animals or legs at that averaged stage: $31 \%$-Pioneer neurons begin axonogenesis; $32 \%$ - the pioneer growth cones are about halfway along the dorsal path (i.e., the site where the Fel cell differentiates); 33\%the pioneer growth cones have completed the dorsal path (i.e., reached the distal border of the coxal segment); $34 \%$-the pioneer axons are at the $\mathrm{Cx} 1$ guidepost cells; $35 \%$ - the pioneer axons have reached the CNS.

\section{Numbers of growth cones examined}

Using the anti-HRP labeling technique, we have examined the morphologies of approximately $5000 \mathrm{Til}$ growth cones (two Tils per leg) in approximately 500 animals from about 50 different clutches of eggs. Using the combined Hoechst/antibody labeling, we have examined several hundred growth concs in animals from over 10 different clutches of eggs.

\section{Selection of clutches with precocious pioneer neuron differentiation (PPD clutches)}

In order to examine the morphologies of pioneer growth cones navigating proximally in the absence of influence by guidepost cells, we exploited two aspects of grasshopper development that we have consistently observed: First, there is up to $2 \%$ variation in the time of differentiation of individual cells and features relative to other cells and features; and second, the variation among eggs within the same clutch of eggs is much lower than variation between clutches. Similar observations concerning consistency within and variations between clutches of grasshopper eggs have been described previously (Goodman et al., 1979; Steeves and Pearson, 1983).

We screened embryos from many clutches to identify clutches where the pioneer neurons differentiate early with respect to the $\mathrm{Fel}$ and $\mathrm{Tr} 1$ guidepost cells; these neurons must extend growth cones without guidance by Fel and $\operatorname{Tr} 1$ (see Results). Such clutches were found and identified as "precocious pioneer differentiation" (PPD) clutches. A clear criterion for PPD clutches is that the Fel and Tr1 guidepost cells label with antibody later than usual (i.e., about the time the Ti1 growth cones reach the CNS), suggesting that those cells probably also differentiate as functional guidepost cells later than usual. Also, in PPD clutches the legs show a lower degree of segmentation than in average clutches (see Results).

The Til growth cone morphologies also can indicate the presence or absence of specific cells and features in the leg. In PPD clutches, the pioneer growth cones generally show no sign of response to the tibiafemur segment boundary (see Results and Fig. 8). Similarly, the characteristic response that growth cones exhibit after contact with guidepost cells can indicate whether the $\mathrm{Fe} 1$ and $\mathrm{Tr} 1$ guidepost cells are present at the time of axonogenesis. Figure $7 \mathrm{~A}$ illustrates the characteristic pioneer morphology in the presence of those guidepost cells. Pioneer contact with guidepost cells typically results in (1) directed reorientations toward guidepost cells, (2) preferential wrapping of those cells with branches and filopodia, and (3) a general lack of branch extension in other directions. These characteristic morphologies can reveal guidepost cells when they are present but unlabeled.

In contrast, growth cones in PPD clutches have profoundly different morphologies (Fig. 7B). These growth cones have several long and prominent branches, and the branches can extend around as much as $90 \%$ of the limb circumference. Such branches are not oriented toward specific sites within the leg and tend to meander more than the highly oriented branches seen in non-PPD clutches. This striking contrast in morphology is the strongest indicator of the absence of differentiated (but unlabeled) guidepost cells. The criteria described above are correlated, and together they allow the unequivocal identification of PPD clutches.
The lack of growth cone response to guidepost cells in PPD clutches is not due to abnormal neuronal differentiation. In older (post 35\%) embryos from PPD clutches, the Fel and Tr1 cells do arise at their normal locations, but later than usual, when the Til growth cones have nearly reached the CNS (Fig. $8 F$ ). The Til neurons apparently respond normally to those cells once they appear: Pioneer filopodia and lateral branches extend preferentially toward and around those cells once they arise (Figs. 2, $A, B$ and $8 F$ ), as occurs in all other clutches. Thus, PPD clutches apparently differ only in the time of Til axonogenesis relative to the differentiation of other cells and features in the leg. The Til growth cones apparently navigate through a normal, but relatively undifferentiated, leg in which the Fe1 and $\operatorname{Tr} 1$ guidepost cells are not yet present.

\section{Quantitation of growth cone branching along the initial path}

Branches of Til growth cones were counted in 20 legs of animals from two PPD clutches, using tracings made from photographs of whole legs (e.g., those in Fig. 8; sample tracings are shown in Fig. 9). The Til neurons each have a single axon, which ends in a growth cone that (in PPD clutches) typically has multiple branches. Branches can be unambiguously distinguished from filopodia in these growth cones. Filopodia are very small $(<0.5 \mu \mathrm{m})$ in diameter and also are extremely uniform in diameter from their tip to within $2-3 \mu \mathrm{m}$ of their base. Branches vary widely in diameter along their length and can be as wide as an axon (about $5 \mu \mathrm{m}$ ). Although branches can be less than $1 \mu \mathrm{m}$ in diameter, they are always larger in diameter than filopodia.

In PPD clutches, leg segmentation is relatively undeveloped at the time of pioneer axonogenesis, and the location of the distal coxal boundary that forms the proximal end of dorsal path must be estimated. This location was estimated using two criteria: the usual position of the boundary relative to the location of the $\mathrm{Cx} 1$ cells in legs where those cells label early ( $\mathrm{T} 1$ and $\mathrm{T} 2$ legs), and by early signs of epithelial constriction at the boundary. The distal end of the dorsal path is defined by the proximal edge of the Til cell bodies.

To quantify branching, each growth cone was first assigned to a region along the dorsal path (see Fig. 9). For each tracing the region containing the greatest number of branches was identified as the "center of growth cone activity" and considered to be the position of the growth cone along the dorsal path. (Branches extended through more than one region were counted in each.) Branch counts from all growth cones occupying the same region were then summed and averaged.

\section{Naming of cells and axes}

In previous studies from this laboratory (Keshishian and Bentley, 1983ac), the early neurons, both guidepost and pioneer, were named according to the apparent segmentation of the early embryonic leg. These cells have now been followed further through development, so that their positions have been determined after complete leg segment differentiation. In order to facilitate comparison with adult legs, and with legs in other insects, the early cells are now renamed according to their positions after all segments have differentiated.

\section{Corresponding names}

$\begin{array}{cllllllll}\begin{array}{c}\text { Previous } \\ \text { name: }\end{array} & \text { CT1 } & \text { CT2 } & \text { F2 } & \text { F4 } & \text { F1 } & \text { F3 } & \text { Ti1 } & \text { Ti2 } \\ \text { New name: } & \text { Cx1 } & \text { Cx2 } & \text { Tr1 } & \text { Tr2 } & \text { Fe1 } & \text { Fe2 } & \text { Ti1 } & \text { Ti2 }\end{array}$

Similarly, the axes of the leg (anterior, posterior, dorsal, and ventral) were initially named with respect to the embryonic body axes. Hereafter, we will describe cell positions with respect to the axes of the adult mesothoracic leg in its normal resting position perpendicular to the adult body.

Corresponding axes

$\begin{array}{ccccc}\begin{array}{c}\text { Axes of embry- } \\ \text { onic body: }\end{array} & \text { anterior } & \text { posterior } & \text { dorsal } & \text { ventral } \\ \begin{array}{c}\text { Axes of adult } \\ \text { (meso) leg: }\end{array} & \text { dorsal } & \text { ventral } & \text { posterior } & \text { anterior }\end{array}$

\section{Results}

\section{Evidence for proximal guidance cues other than} guidepost cells

The Til pioneer cells arise as a sibling pair of peripheral neurons. At about the $30 \%$ stage of embryonic development, they dif- 

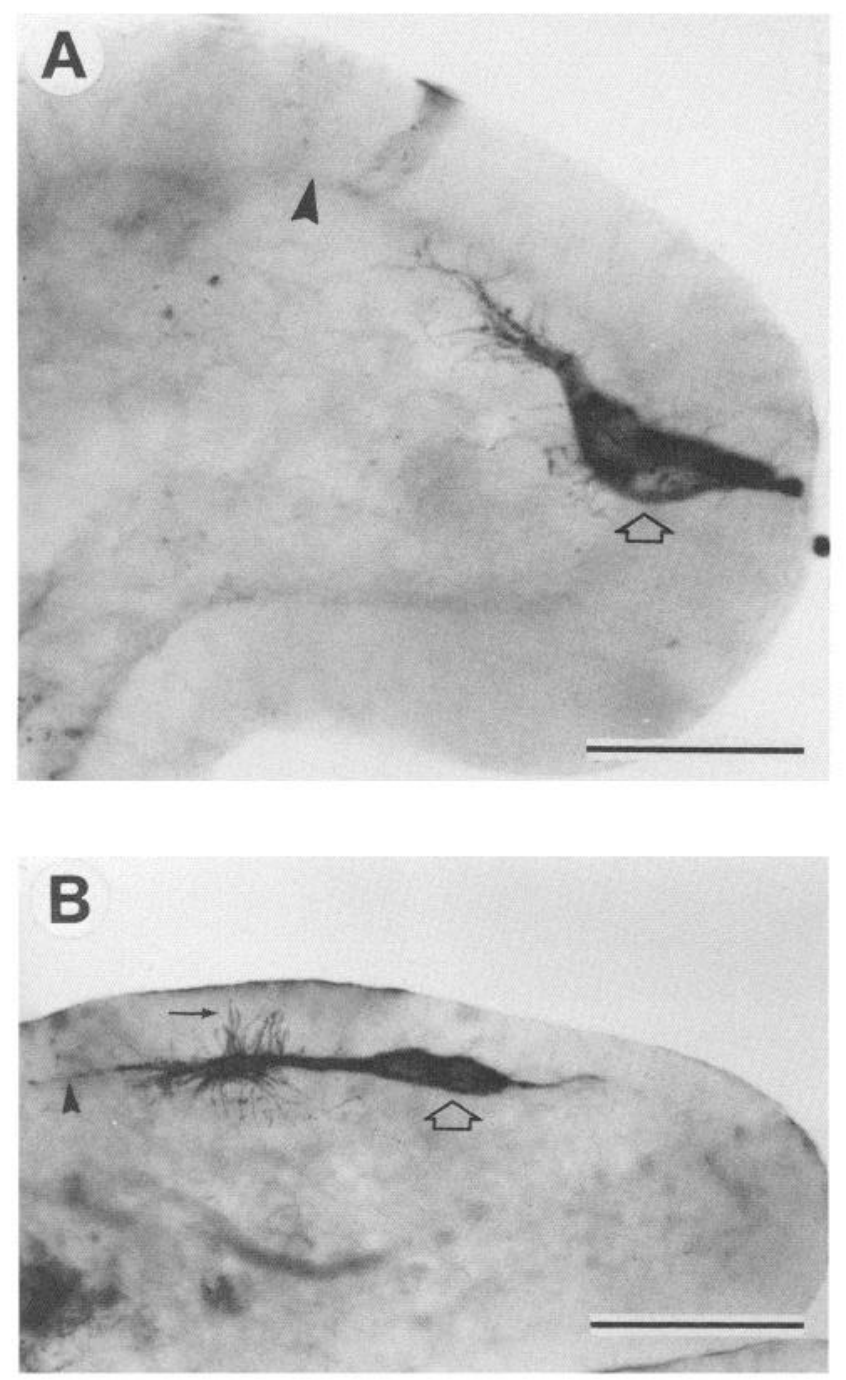

Figure 1. Til pioneer neuron axonogenesis. $A$, At the $31 \%$ stage, a pair of sibling pioneer neurons (open arrow) differentiate within the epithelium at the tip of the leg and then emerge from the epithelium and lie on its inner surface (carat). Axons grow proximally from the cell bodies and can do so before the cells have fully emerged from the epithelium. Calibration bar, $50 \mu \mathrm{m}$. B , Pioneer growth cones grow along the inner surface of the epithelium. Filopodia are extended into the lumen and contact mesodermal cells there. Many filopodia (arrow) also extend into the spaces between epithelial cells and can reach to within a few microns of the outer surface of the epithelium. The pioneer growth cones and branches remain on the inner surface of the epithelial substrate and often follow leading filopodia there (carat). Calibration bar, 100 $\mu \mathrm{m}$.

ferentiate from the ectodermal epithelium at the tip of the leg and emerge from the epithelium to lie on its inner surface (Fig. $1 A)$. Following an initial period of apparently random filopodial extension, growth cones emerge from the proximal poles of the cell bodies and grow proximally on the inner surface of the ectodermal epithelium (Fig. $1 B$ ). Proximal growth is continued until the growth cones have navigated through the entire femur and reach the distal segment boundary of the coxa. Here, they make the first of two characteristic turns on their route to the CNS. The initial region of proximally directed growth will be referred to as the "dorsal path." (Refer to Fig. 12, 35-42\% stage leg, for a diagram of the completed Til path, and the location of the various guidepost cells along it. In the following, the term "pioneer" will refer to the Til pioneers unless otherwise specified.)
As they navigate on the inner epithelial surface, the Til growth cones extend many filopodia into the interstitial spaces between epithelial cells (Fig. $1 B$ ). These filopodia can reach to within $2-$ $3 \mu \mathrm{m}$ of the outer epithelial surface. Filopodia are also extended into the limb lumen. They contact loosely packed mesodermal cells (Fig. $1 B$ ) and can be as long as those extended within the epithelium. However, growth cone branches and the pioneer axons extend only along the inner surface of the epithelial substrate, often following long leading filopodia along that surface (Fig. 1B). Thus, pioneer growth cones exhibit a clear preference for the inner epithelial surface over either mesodermal cells or ectodermal cell surfaces within the epithelium.

Prominent guidance cues for the Til growth cones are a set of immature neurons whose axonless cell bodies lie at about 40 $\mu \mathrm{m}$ intervals on the epithelial surface and that apparently act as "guideposts" toward which growth cones orient (Bentley and Keshishian, 1982a, b; Ho and Goodman, 1982). The Fel and Tr1 guideposts lie proximal to the Til cell bodies, on the dorsal path. Filopodial contact with either of these guidepost cells apparently causes pioneer growth cones to reorient distinctly toward and grow directly to them (Fig. $7 \mathrm{~A}$; also discussed in more detail in M. Caudy and D. Bentley, unpublished observations).

However, embryos are occasionally found in which the pioneer axons extend straight proximally from their cell bodies but do not pass over the Fel cell (Fig. 2, $A, B$ ). Lateral pioneer filopodia and branches often still extend to that cell, but the pioneer axons do not directly contact it.

Similarly, pioneer axons are sometimes seen that have completed proximal growth to the distal coxal boundary without cuing on the $\operatorname{Tr} 1$ guidepost. This is clearest in legs in which the pioneer growth cones have followed separate proximal routes to the segment boundary, at which point they reorient circumferentially (Fig. 2, $C, D$ ). Although each has grown proximally, both cannot have cued on a single $\operatorname{Tr} 1$ guidepost cell. Furthermore, the axons do not merely emerge proximally from their cell bodies and continue in the same direction, but sometimes reorient proximally after deviating from proximal growth (Fig. $2 C$; also see Bentley and Caudy, 1983b).

Therefore, although the Fe1 and Tr1 cells typically serve as proximal guidance cues, the pioneer growth cones can still navigate proximally without orienting toward those cells. These observations suggest the presence of an additional proximal guidance mechanism.

\section{Changes in growth cone morphology and orientation at limb segment boundaries}

\section{Increased branching, spreading, and filopodia at the tibia-femur segment boundary}

At the time of axonogenesis, the pioneer cell bodies usually lie between 10-20 $\mu \mathrm{m}$ distal to the tibia-femur segment boundary. Occasionally they lie further proximally, and sometimes they are positioned directly on the segment boundary. The segment boundary is clearly evident by the $42 \%$ stage as a prominent constriction in the leg epithelium (Fig. 5, $A, B$ ), and this constriction begins to appear at about the $35 \%$ stage.

Pioneer growth cones exhibit three changes in morphology and behavior as they cross the tibia-femur segment boundary: (1) extension of lateral branches, (2) spreading of lateral lamellae, and (3) prolonged retention (or extension) of lateral filopodia. The first response observed is the extension of lateral branches and filopodia along the segment boundary (Fig. $3 \mathrm{~A}$ ). Lateral branches can be extended circumferentially in either direction or both. However, these lateral branches are apparently withdrawn during development, as pioneer neurons at later stages no longer have them (Fig. 3, $C, D$ ). The most common response is lateral spreading of lamellae from pioneer axons whose growth cones have already crossed the boundary (Figs. 

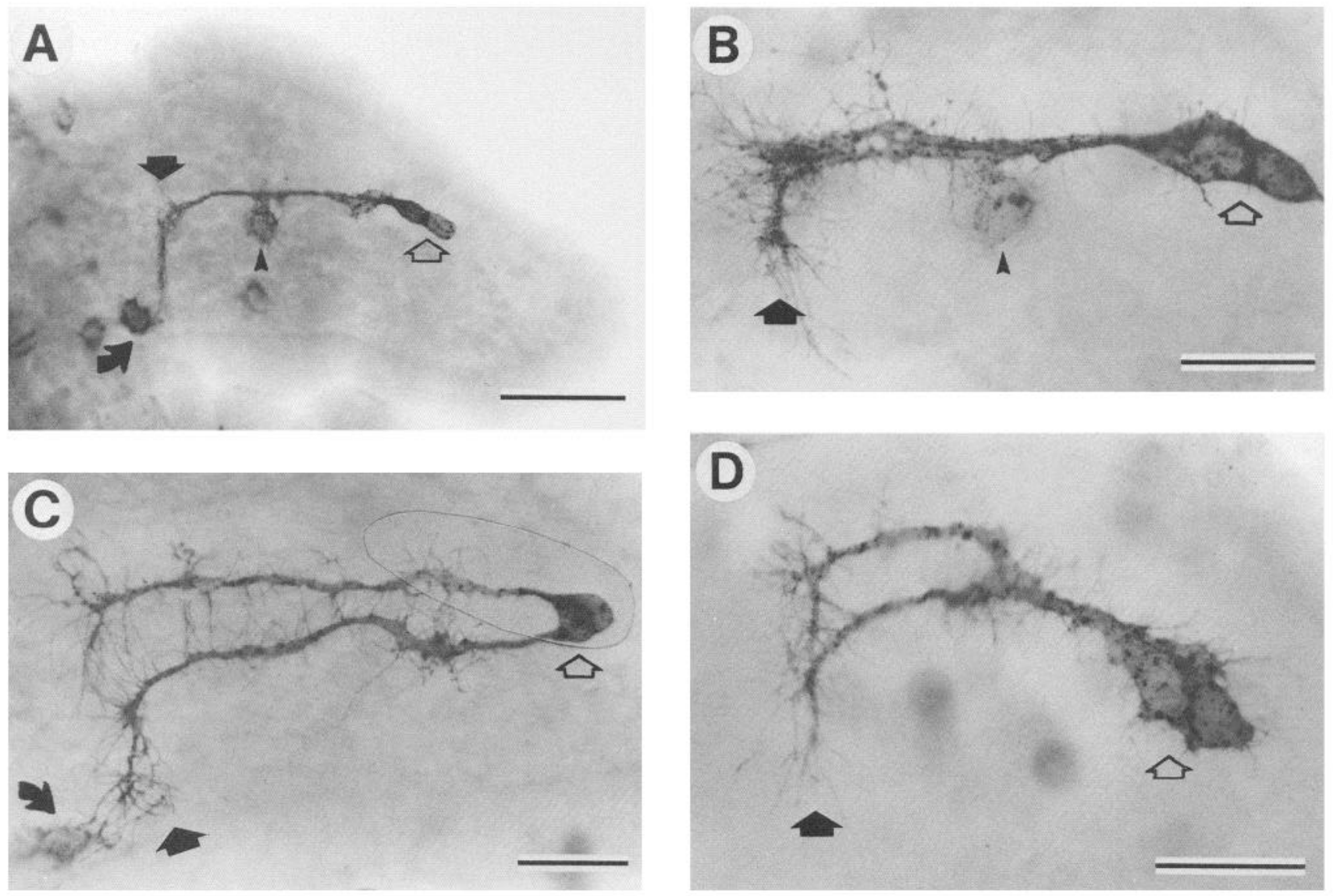

Figure 2. Proximal Til axonogenesis without guidepost cell contact. $A$, The pioneer neurons (open arrow) have axons that extend along the limb axis. They reorient and grow ventrally around the limb circumference along the distal coxal segment boundary (solid arrow) and indirectly toward the Cxl guidepost cell pair (curved arrow). The axons do not contact the Fe1 guidepost neuron (carat). Development stage, $34 \%$. Calibration bar, $100 \mu \mathrm{m}$. B. Higher magnification view of similar behavior in a different limb. Although the axons themselves do not contact the Fel cell (carat), lateral filopodia have been extended that do contact it. Open arrow, pioneer cell bodies; solid arrow, distal coxal segment boundary. Calibration bar, $50 \mu \mathrm{m}$. $C$, Each pioneer neuron (open arrow) has extended an axon on a separate route along the limb axis. The axons contact the distal coxal segment boundary (solid arrow) at widely separated points. This indicates that both axons cannot have been guided by a single (unlabeled) Tr1 guidepost cell. The Til axons have navigated proximally through the complete dorsal path region, despite occasional reorientations away from proximal growth, suggesting that an initial intrinsic polarity cannot account for all of their proximal guidance. Curved arrow, Cx1 cells. Calibration bar, $50 \mu \mathrm{m}$. $D$. A similar occurrence in a different leg at a slightly later stage of pioneer growth. The separated axons have each distinctly reoriented circumferentially at the segment boundary (solid arrow). Open arrow, pioneer cell bodies. Calibration bar, $50 \mu \mathrm{m}$.

$2 A, 3 B, 5 C$, and $7 A$ ). These lateral lamellae are often extended in both directions at the same axial location (Fig. $3 B$ ). They usually have a characteristic triangular shape with prominent points at the boundary and are generally about $20 \mu \mathrm{m}$ wide at the base (Fig. 3, $B, C$ ). Such lamellae persist at this location and can be observed to lie along the epithelial constriction that marks the segment boundary at later stages (Fig. 3C). The lateral lamellae and branches occur at varying distances from the Til cell bodies (cf. Figs. $3 C$ and $11 A$ ) and can arise directly from the cell bodies, if they are located at the segment boundary (Fig. $11 A)$.

Lateral filopodia are more prevalent at the segment boundary (Fig. $3 D$ ) and are also generally longer than filopodia extended from nearby locations. The prevalence of filopodia occurs in a region about the same width as the lamellae observed at the segment boundary, $20 \mu \mathrm{m}$, suggesting that the cue at the segment boundary is wider than a single circumferential line. The filopodia at the boundary persist despite the general tendency for filopodial withdrawal along the axon as the growth cone advances further.

The neuronal response to the boundary does not appear to be due to a physical barrier there. Examination of the region with Nomarski optics and with stained LM sections (data not shown) did not reveal a ridge or groove on the inner epithelial surface. Also, antibody-labeled lamellae appear to lie flat within a very narrow $(<0.5 \mu \mathrm{m})$ plane of focus. If there is, in fact, no morphological feature there, these observations could be due to the presence of a chemical change in surface properties of the epithelium. This would be consistent with observations in culture, where growth cone branching, lamellar extensions, and prolonged filopodial retention are all characteristics of growth cones traversing highly adhesive substrates (Letourneau, 1975a, 1979).

\section{Growth cone reorientation at the distal segment boundary} of the coxa

After crossing the tibia-femur segment boundary, the pioneer growth cones continue to grow proximally until they encounter the distal boundary of the coxa. It is at this boundary that the growth cones abruptly reorient circumferentially and make their first characteristic turn (Fig. 4; Bentley and Caudy, 1983b). This boundary eventually separates the coxa and trochanter, but when the Til growth cones contact it at the 33\% stage, the trochanteral segment is not distinguishable from the proximal region of the femur. The pioneer growth cones usually show no response to the femur-trochanter segment boundary until later in devel- 

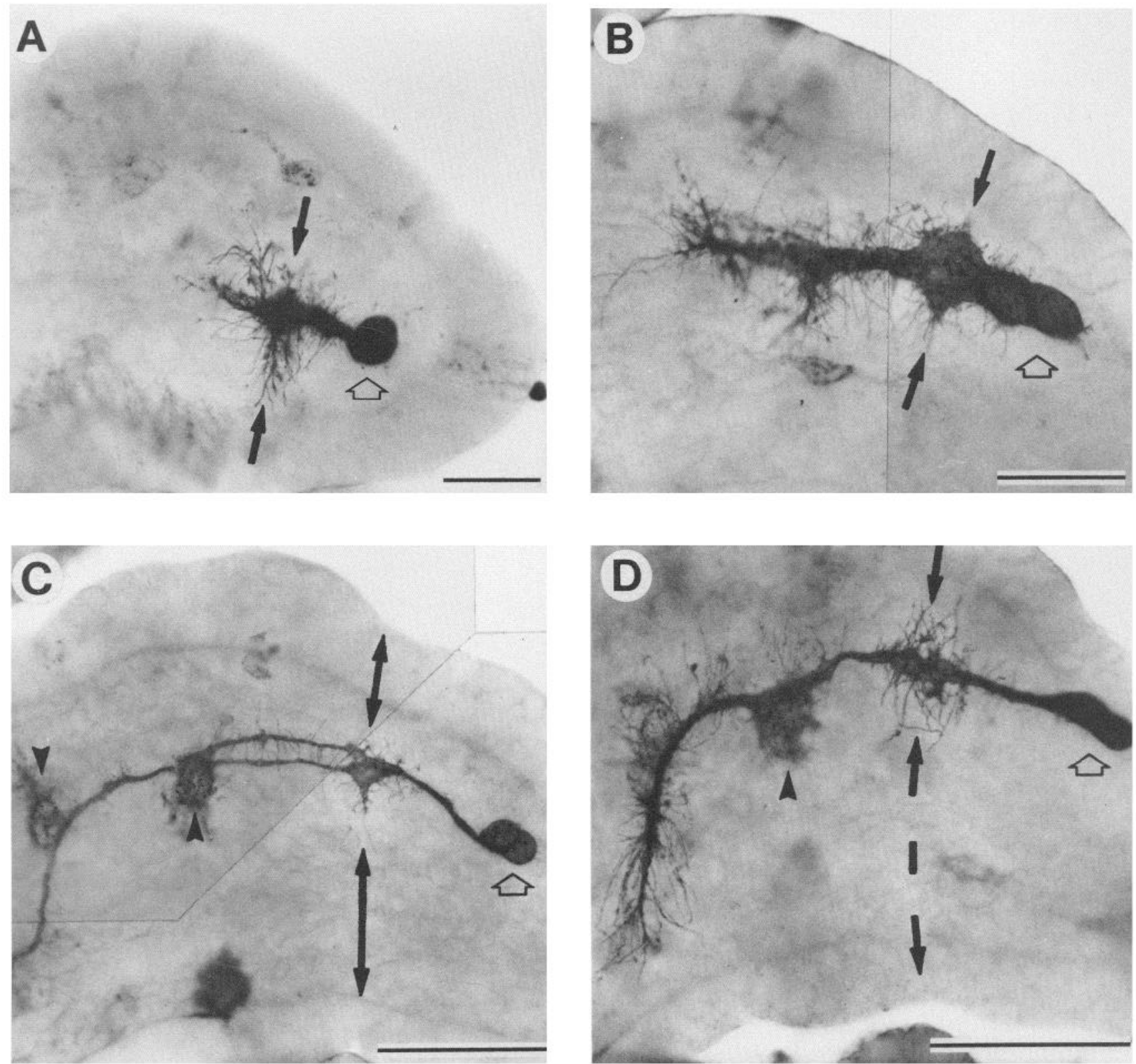

Figure 3. Pioneer growth cone morphology at the tibia-femur segment boundary. A, At the late $31 \%$ stage, pioneer neurons (open arrow) have extended growth cones proximally. A prominent branch and many lateral filopodia are extended circumferentially along the tibia-femur segment boundary (arrows). Calibration bar, $50 \mu \mathrm{m}$. B. At the $32 \%$ stage, growth cones from the Til cells (open arrow) have grown proximally across the tibia-femur segment boundary (solid arrows). Characteristic triangular lamellae have spread in both directions at the boundary. Calibration bar, $50 \mu \mathrm{m}$. C, The epithelial constriction that morphologically marks the tibia-femur boundary (solid arrows) is evident in a somewhat older ( $35 \%$ stage) leg. The pioneer axons are flattened where they cross the segment boundary and have extended a triangular lamellum ventrally along it. Downward carat, Tr1 cell; upward carat, Fe1 cell; open arrow, pioneer cell bodies. Calibration bar, $100 \mu \mathrm{m}$. D, At about the $34 \%$ stage, filopodia extended from the pioneer axons have been preferentially retained (or extended) where they cross the segment boundary (arrows). Filopodia in adjacent regions, both proximally and distally, have been retracted. Upward carat, Fe1 cell; open arrow, pioneer cell bodies. Calibration bar, $100 \mu \mathrm{m}$.

opment. By the $42 \%$ stage, both the proximal and distal boundaries of the trochanter are clearly evident in Hoechst-labeled legs (Fig. 5A).

The epithelium in this region is also constricted, and this constriction is normally evident by the $33-34 \%$ stage of development (Fig. 2A). Thus, at the stage when the pioneer growth cones contact it, the distal coxal boundary appears to be more fully differentiated than was the tibia-femur boundary.

As at the tibia-femur boundary, when the pioneer growth cones contact the distal coxal boundary, they extend branches circumferentially along it (Fig. 4A). However, at this boundary, further axial growth is arrested, and the growth cones and axons themselves reorient circumferentially along the boundary. Initially, branches are often extended circumferentially for equal distances in both directions (Fig. $4 A$ ). Eventually, the ventrally directed branch predominates and the axons turn in this direction (Fig. $4 B$ ). This turn appears to depend on filopodial contact with the proximoventrally located $\mathrm{Cx} 1$ guidepost cells (Bentley and Caudy, 1983b).

Growth cones on the segment boundary often follow a very straight line on the epithelial substrate (Fig. $4 B$ ). Branches do not extend either proximal or distal to the boundary (Fig. 2, $A$, $B, D$ ), except along filopodia in contact with the $\mathrm{Cx} 1$ guidepost cells (Fig. 4B). However, broad lamellae are occasionally ob- 

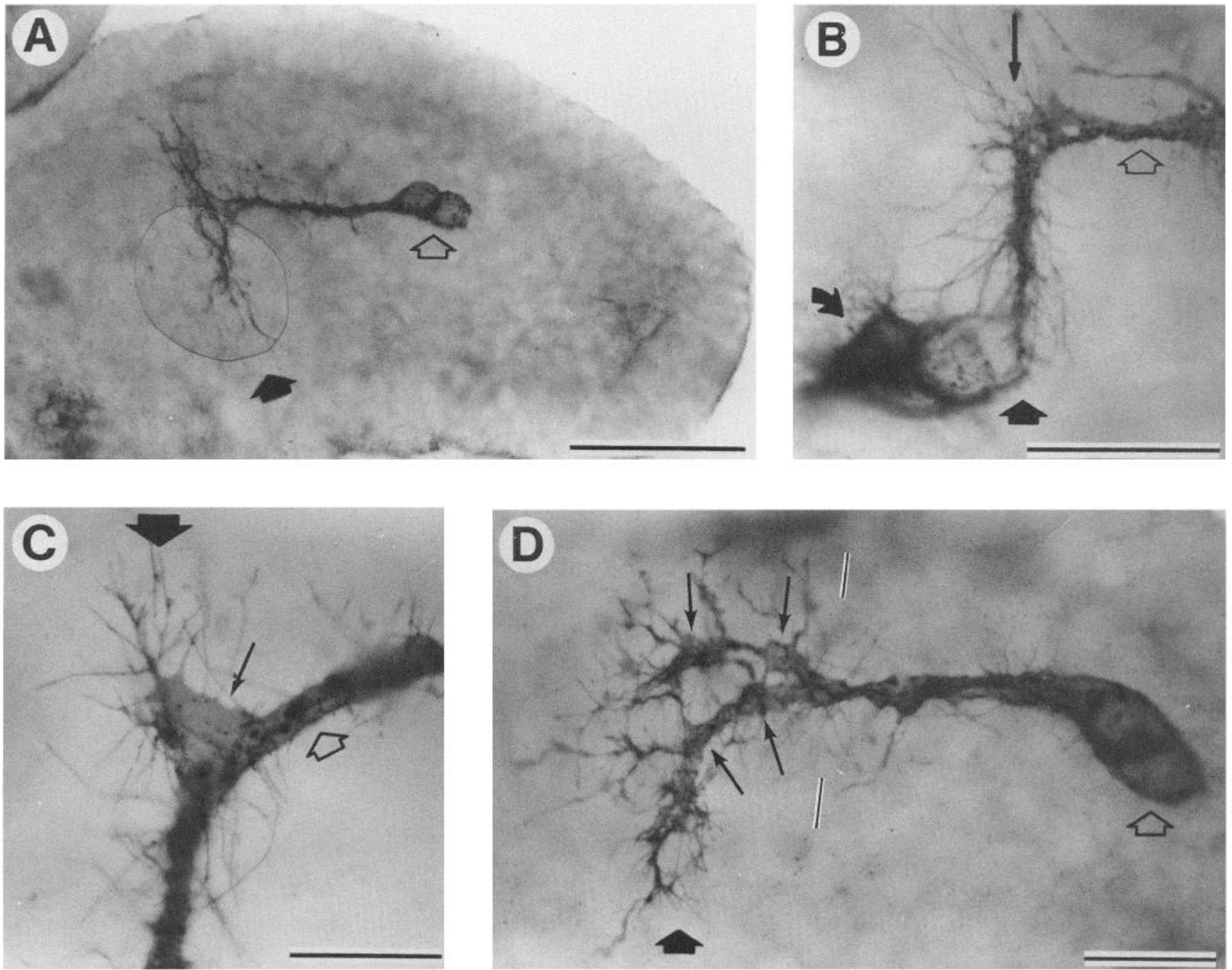

Figure 4. Pioneer growth cone morphology at the distal coxal boundary. $A$, The pioneer axons cease proximal growth when they encounter the distal boundary of the coxa (solid arrow) and often extend processes in both directions around the limb circumference. Open arrow, pioneer cell bodies. Calibration bar, $100 \mu \mathrm{m}$. B. Subsequently, the pioneer axons often turn along a very sharp line (long arrow) at the segment boundary (solid arrow). Pioneer filopodia extend further proximally, but branches do not extend proximal to the segment boundary except along filopodia which have contacted the proximally located $\mathrm{Cx} 1$ guidepost cells (curved arrow). Open arrow, pioneer axons. Calibration bar, $50 \mu \mathrm{m}$. $C$, The pioneer axons occasionally spread broad lamellae (long arrow) in the region just distal to the segment boundary (solid arrow), between the Til axons (open arrow) and a branch extended dorsally along the segment boundary. Such lamellae are very flat and therefore do not appear to be spread over an unlabeled $\operatorname{Tr} 1$ cell at this location. Calibration bar, $50 \mu \mathrm{m}$. $D$, In the proximal region of the initial path, the pioneer growth cones flatten and spread lamellae (small arrows) on the epipthelium. Open arrow, pioneer cell bodies; black and white lines mark the halfway point through the femur (see text). Calibration bar, $50 \mu \mathrm{m}$.

served at, and just distal to, the segment boundary (Fig. 4C). In contrast to branches, filopodia readily extend both proximally and distally to the boundary (Figs. 2 and $4 B$ ).

Thus, at both the tibia-femur segment boundary and the distal coxal boundary, pioneer growth cones appear to encounter a narrow circumferential band on the epithelial substrate that influences their morphology and orientation. As at the tibiafemur boundary, no morphological features are evident at the LM level that might act as physical barriers to further proximal growth.

\section{Asymmetries in axon branching and spreading on opposite sides of segment boundaries}

\section{Asymmetries at the tibia-femur boundary}

Differential response to the epithelium in the tibia-femur boundary region is initially localized to the boundary. When the lamellae are first observed at the boundary (at the $32 \%$ stage), branching or spreading of the pioneer axons is not observed either proximally or distally (Fig. $3 B$ ). However, later in development (the $35 \%$ stage; Fig. $5 C$ ), the pioneer axons flatten and spread in the region distal to the boundary (proximal tibia) but not in the region proximal to it (distal femur). The region of axon in the proximal tibia can spread to more than twice the width of the region of axon in the distal femur.

This regional difference in apposition of the neuron to the epithelial substrate increases with development. By the $42 \%$ stage, the pioneer axons have developed pronounced lateral branching in the proximal tibia (Fig. 5, $B, D$ ). These branches can extend $20-30 \mu \mathrm{m}$ to either side of the axon. Figure 6 shows tracings of the branching patterns of three pioneer axon pairs in the segment boundary region.

In contrast, the length of axon within the distal femur exhibits no tendency to spread or branch on the epithelium as development proceeds (Figs. 5, $C, D$ and 6). That region of axon also has few or no lateral filopodia. 

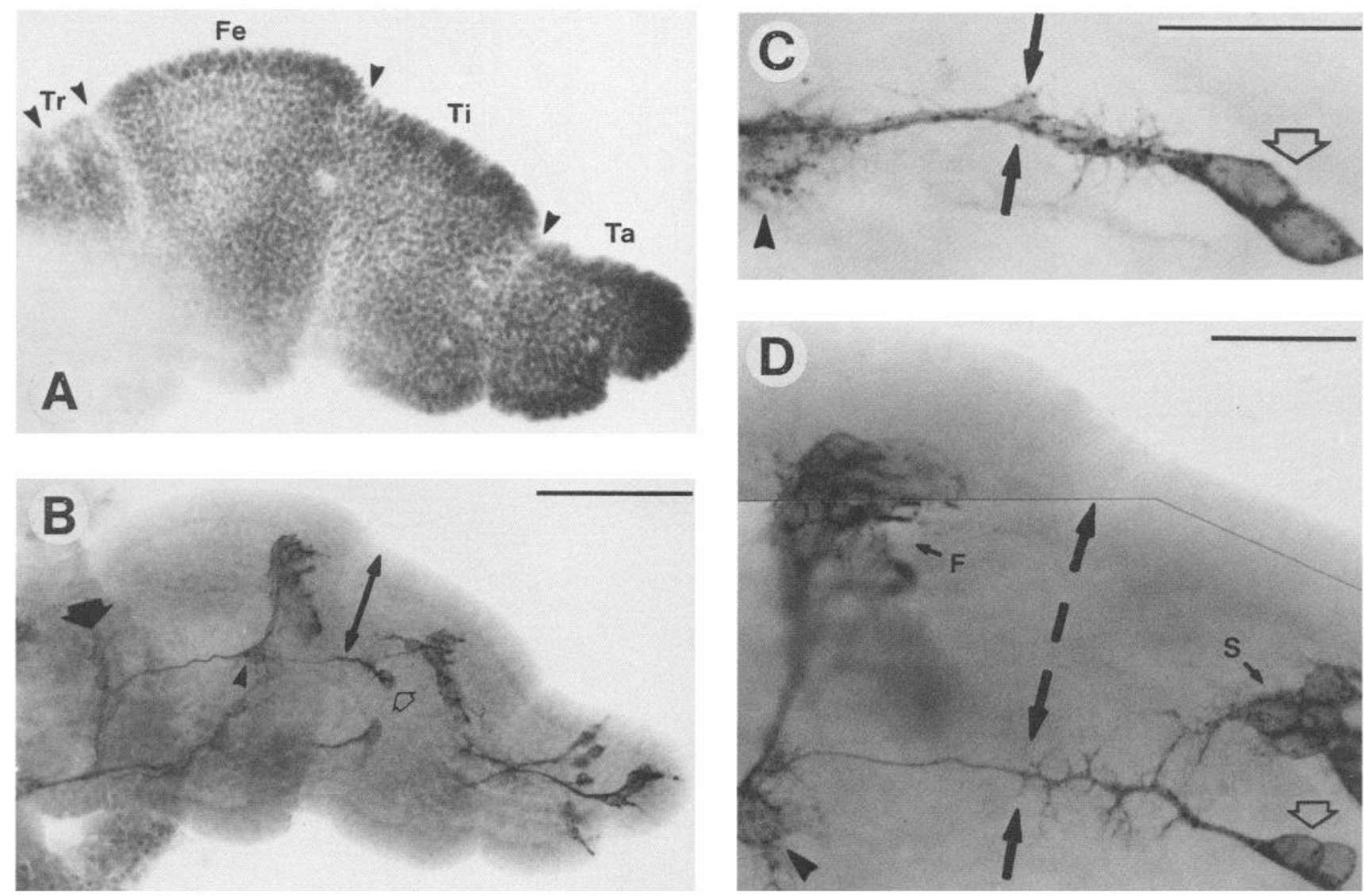

Figure 5. Pioneer morphologies on opposite sides of the tibia-femur boundary. $A$, At the $42 \%$ stage, the trochanter ( $T r$ ), femur $(F e)$, tibia (Ti), and tarsus $(\mathrm{Ta})$ are evident in legs labeled with the nuclear stain, Hoechst 33258 . The boundaries between these segments are apparent as changes in the contour of the epithelium (carats) and as alterations in nuclear spacing. Calibration bar $(A, B), 200 \mu \mathrm{m}$. $B$, The same leg as in $A$, labeled with anti-HRP antibodies to show neural development at this stage. The pioneer neurons (open arrow) lie distal to the tibia-femur boundary (double-headed arrow). The pioneer axons have extended lateral branches in the proximal region of the tibia but have no lateral branches in the distal region of the femur. Tr1 and the circumferential path are located at the trochanter-coxa segment boundary (solid, broad arrow). Upward carat, $\mathrm{Fe} 1$ cell. $C$, At the $35 \%$ stage, lateral lamellae of the pioneer axon remain at the segment boundary (solid arrows). The axons are now flattened and spread on the epithelium throughout the proximal region of the tibia, between the Til cell bodies (open arrow) and the segment boundary. However, the axons are not flattened in the distal region of the femur, between the tibia-femur segment boundary and the Fel cell (carat). Calibration bar, $50 \mu \mathrm{m}$. $D$, A different $42 \%$ stage leg at higher magnification than in $B$. In addition to flattening, the pioneer axons have extended many secondary branches laterally in the proximal tibia. In contrast, they have no lateral branches or filopodia in the distal femur, and the axons are not spread in that region. $F$, femoral chordotonal organ; $S$, subgenual organ; open arrow, pioneer cell bodies; solid arrows, tibia-femur segment boundary; carat, cell Fe1. Calibration bar, $50 \mu \mathrm{m}$.

Thus, neuronal morphologies are distinctly different in the region proximal to the tibia-femur segment boundary versus the region distal to it. Growth of the same pioneer axon through both regions allows a direct comparison between neuronal responses to the substrate in them and directly reveals the asymmetry.

\section{Asymmetries at the distal boundary of the coxa}

No such direct comparison is possible for the epithelial regions adjacent to the distal coxal boundary, since the pioneer axons do not cross that boundary when they first encounter it. (When they eventually cross to the $\mathrm{Cx} 1$ guidepost cells, contact with those cells appears to strongly influence growth cone morphology.) However, the failure of pioneer branches and growth cones to enter the distal region of the coxa without $\mathrm{Cx} 1$ contact suggests an affinity for that region that is lower than that for other available substrates. In contrast, the pioneer growth cones have a pronounced tendency to branch and spread in the proximal region of the femur, as they approach the distal coxal boundary (see below; Figs. $4 D, 8,9$, and 10). These differences in branch extension suggest a higher affinity to proximal femur than to distal coxa.

\section{Differences in pioneer morphology within a single segment}

\section{Precocious pioneer differentiation clutches}

The above observations are consistent with a model in which neuronal affinity for the epithelial substrate increases proximally within a segment, with a peak at the boundary. Such affinity differences within the femur (which comprises the majority of the dorsal path) might explain proximal guidance along the dorsal path in the absence of guidepost cell contact. To investigate this possibility, we have compared neuronal morphologies in the proximal and distal regions of the femur.

A potential problem with this approach is that contact with guidepost cells might mask or override growth cone response to less dominant cues on the epithelium. To avoid the influence of guidepost cell contact on pioneer morphology, we have exploited the normal range of variation in the time of pioneer differentiation relative to the Fel and Trl cells. We screened for clutches in which the pioneers consistently differentiate earlier than usual in the leg, and we have successfully identified such PPD clutches (see Methods).

In PPD clutches, the pioneer growth cones apparently grow through a normal but relatively undifferentiated leg in which 


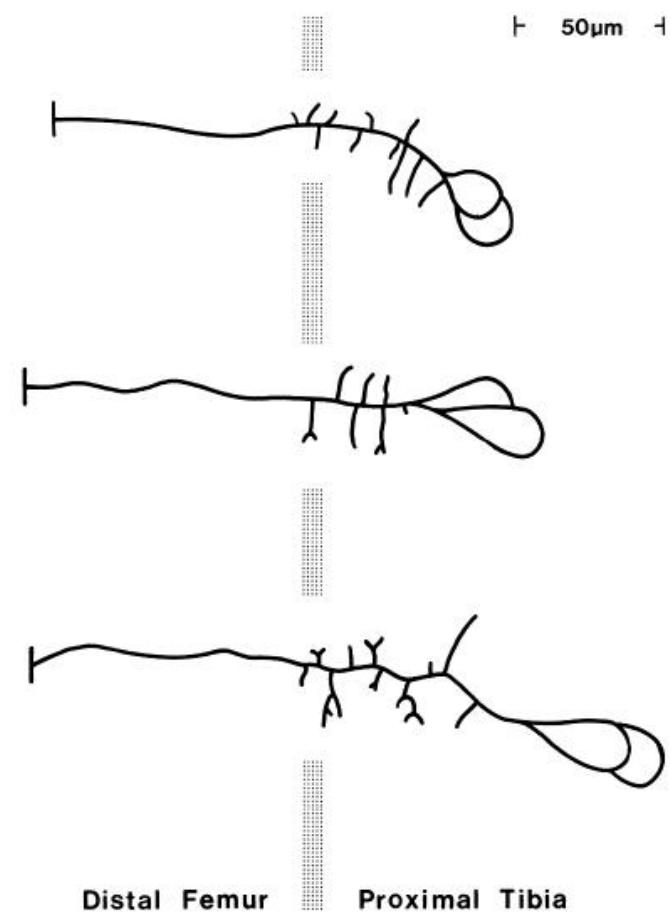

Figure 6. Differences in degree of pioneer branching proximal and distal to the tibia-femur segment boundary. Tracings were made of antiHRP stained pioneer axons and branches in the proximal tibia and distal femur regions between the pioneer cell bodies (at right) and the site where the pioneer axons meet cell Fel (short, vertical lines at left). Filopodia were not traced. Tracings are from three different embryos at the $42 \%$ stage. In each leg, multiple lateral branches have been extended from the Til axons in the proximal region of the tibia. Lateral branching has occurred up to the tibia-femur segment boundary (vertical, stippled bars at center) but has abruptly discontinued there. No lateral branches are extended in the distal region of the femur, which lies proximal to the boundary.

the Fel and Tr1 guidepost cells are not yet present. The Fe1 and $\operatorname{Tr} 1$ cells arise in their normal positions in PPD clutches but later than usual relative to Til differentiation (Fig. $8 F$ and Methods).

There usually is a profound difference in Til morphologies between PPD and non-PPD clutches (see Methods). In nonPPD clutches, the Til growth cones generally exhibit very little, if any, branching so that they are highly oriented in a specific direction. Furthermore, they frequently make a single, distinct reorientation toward either labeled guidepost cells (Fig. $7 A$ ) or the sites where those cells eventually do label with antibody. In contrast, in PPD clutches the Til growth cones generally have multiple branches, which can spread over as much as $90^{\circ}$ of the limb circumference and which do not reorient toward guidepost cells or the sites where those cells will arise (Fig. $7 B$ ).

Similarly, in PPD clutches the Til growth cones generally show no response to the tibia-femur segment boundary (Fig. 8) and apparently navigate proximally along the dorsal path before that boundary has differentiated sufficiently to influence their morphology. Thus, the Til growth cones in PPD clutches apparently navigate proximally through a leg that is relatively less differentiated than in non-PPD clutches, both with regard to specific identified cells and to segment boundaries.

Despite the absence of known extrinsic cues, the Til growth cones still initiate and maintain proximal growth along the dorsal path in PPD clutches.

Growth cone branching increases proximally within the femur PPD clutches provide an opportunity for analyzing the guidance mechanism that directs axons proximally whenever they are not
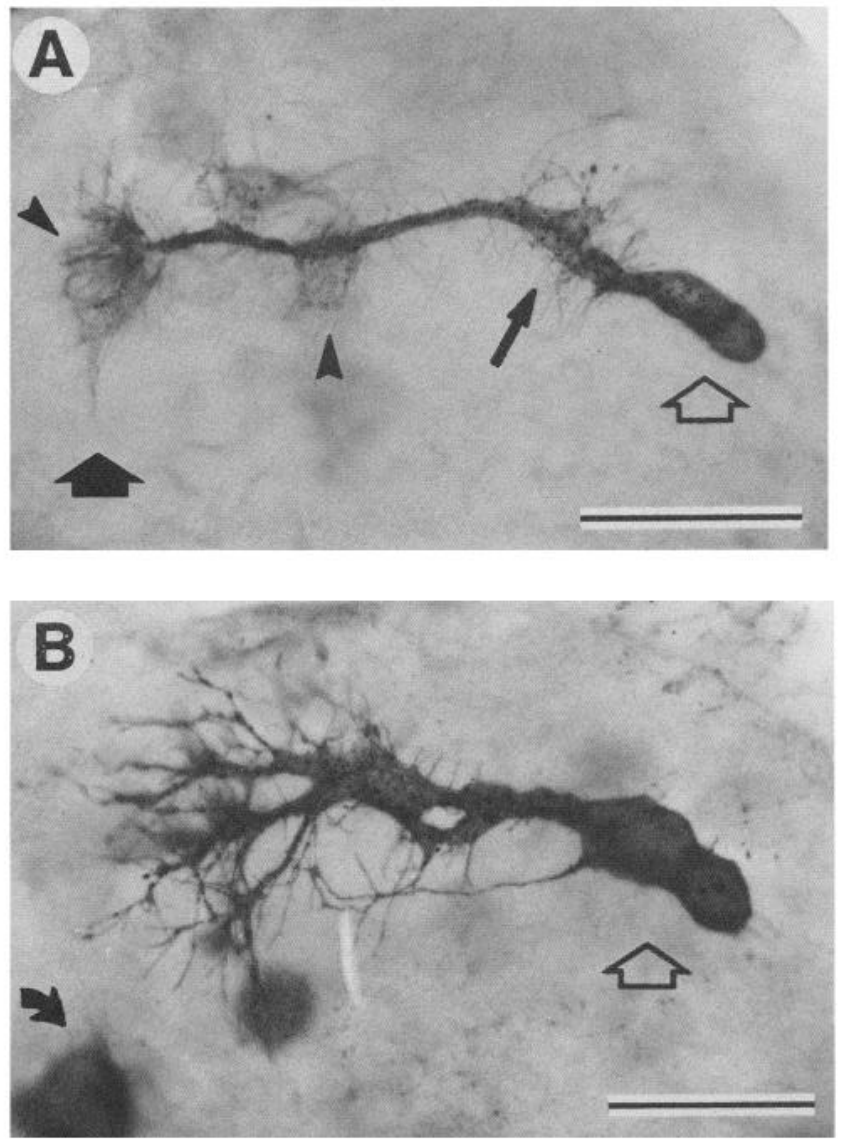

Figure 7. Pioneer growth cone morphology in the presence and absence of guidepost neurons. $A$, In a leg from a non-PPD clutch embryo (see text), the pioneer axons have crossed the tibia-femur segment boundary (long arrow) and then distinctly reoriented toward, and grown to contact, the labeled Fel guidepost (upward carat). After leaving Fe1, they have again grown proximally before again reorienting and growing to the labeled Trl guidepost cell (downward carat). The growth cone and filopodia are spreading across this cell. The Trl cell is on the distal coxal boundary (solid arrow) and has a ventrally oriented growth cone. Note that the pioneer axons have no lateral branches. Open arrow, pioneer cell bodies. Calibration bar, $100 \mu \mathrm{m}$. B, In a leg from a PPD clutch embryo, the pioneer growth cones have also reached the proximal end of the dorsal path, which lies about $20-25 \mu \mathrm{m}$ distal (as well as dorsal) to the $\mathrm{Cx} 1$ guideposts (curved arrow). However, the growth cone morphology is profoundly different from that in $A$. The pioneer axons have many lateral branches, and the branches spread across a broad circumferential arc of the epithelial surface. Note that there are no signs of branch or filopodial response to guidepost cells or to the tibia-femur segment boundary. Open arrow, pioneer cell bodies. Calibration bar, $50 \mu \mathrm{m}$.

in contact with guidepost cells (Fig. 2, $A, B$ ). A series of growth cones from two PPD clutches, at increasingly proximal positions along the dorsal path is shown in Figure 8. These growth cones appear to undergo an abrupt increase in branching at about the midpoint of the dorsal path (Fig. 9). To analyze this phenomenon, we have quantitated branching differences along the dorsal path (see Methods).

While most of the dorsal path lies within the femur, its distal end may include a small region of the tibia, and its proximal end includes the prospective trochanter (which may have begun to differentiate). Therefore, these regions may include branching differences that are not strictly within the femoral segment. Also, the proximal region includes the distal coxal segment boundary, which is known to be a high-affinity cue. Contact with this boundary may influence the degree of growth cone branching 

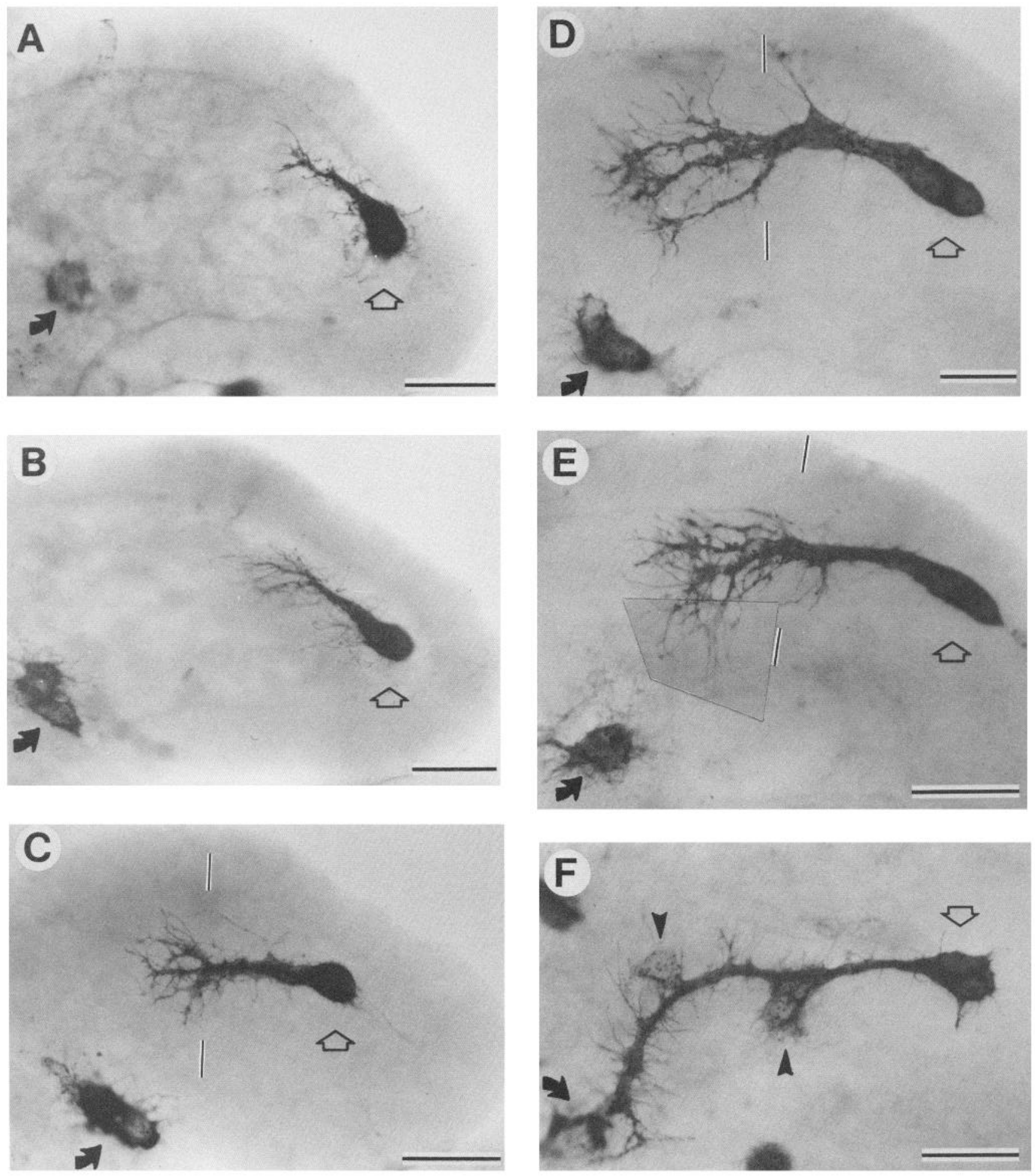

Figure 8. Pioneer growth cone morphologies in PPD clutch embryos at different stages of navigation along the dorsal path. $A$, The pioneer growth cones have extended about $50 \mu \mathrm{m}$ proximally from the Til cell bodies. The growth cones have no lateral branches and are very narrow compared with the width of the cell bodies ( $31 \%$ stage). $B$, The pioneer growth cones have extended about $75 \mu \mathrm{m}$ proximally along the limb axis (parallel to the edge of the leg). The growth cones still are unbranched (late $31 \%$ stage). $C$, The pioneer growth cones have reached the midpoint of the femur (black and white lines) and have begun to branch. They are not in contact with the distal segment boundary of the coxa, which lies just distal to the $\mathrm{Cx} 1$ cells (late $32 \%$ stage). $D$, The two pioneer growth cones have prominent branches in the region of the dorsal path proximal to the midpoint of the femur (black and white lines). Almost no branching occurs distal to that point. Three major branches are just contacting the distal coxal segment boundary ( $33 \%$ stage). E, A high degree of branching occurs in the region proximal to the midpoint of the femur (black and white lines), with only one small branch distal to it (later $33 \%$ stage). F. The pioneer axons have contacted the Cx1 guidepost cells. Fe1 (upward carat) and Tr1 (downward carat) have differentiated and are labeled with anti-HRP. Note that the pioneer axons do not directly contact these cells, although lateral processes do (34\% stage). Legs were chosen from two different PPD clutches. Symbols for all legs: open arrows, pioneer cell bodies; curved arrows, Cx1 cells. Calibration bars, $50 \mu \mathrm{m}$. 
A
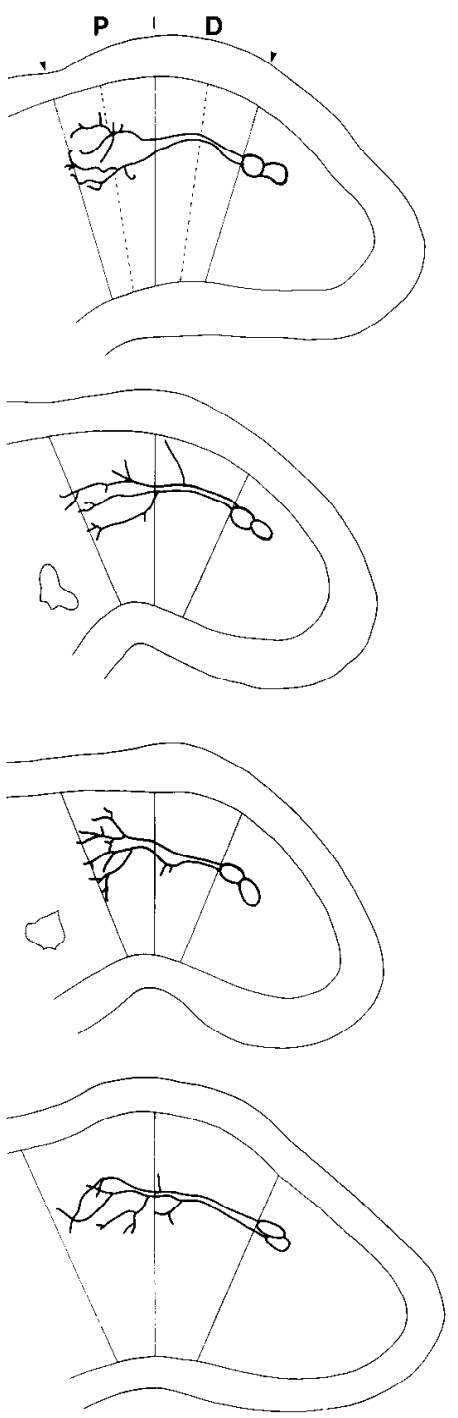

B
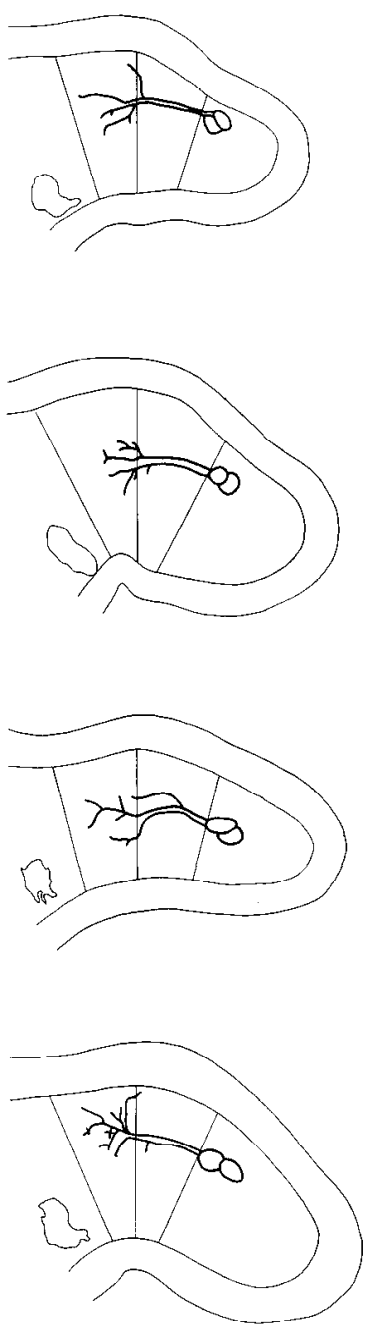

C
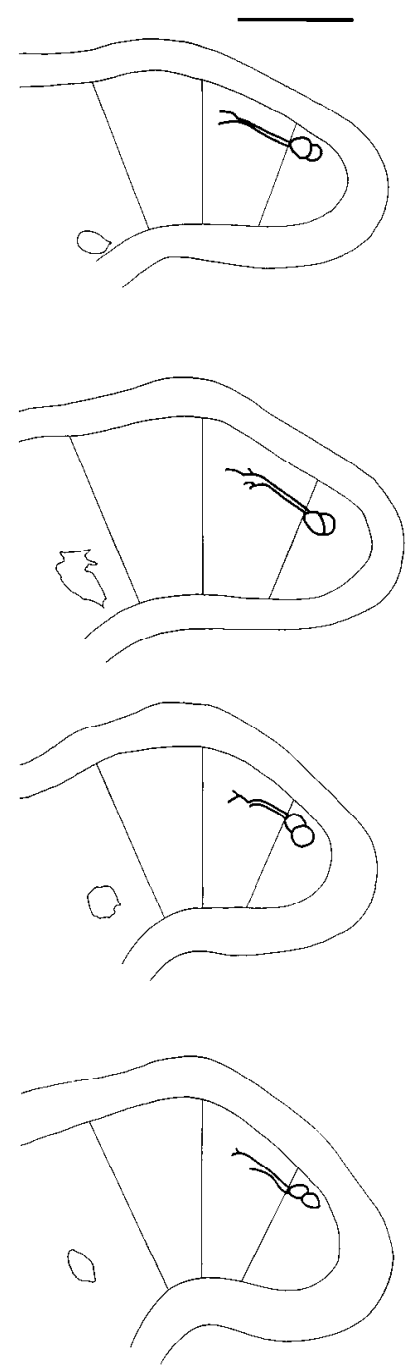

Figure 9. Differential branching of pioneer growth cones in different regions of the dorsal path. A representative set of 12 tracings of pioneer axons and branches (but not filopodia) and limb outlines from PPD clutch embryos used for branch quantitation. In each tracing, the dorsal path region was identified (see Methods) and divided into four equal-sized regions, as shown in the upper-left tracing. The legs have been grouped according to whether their growth cones were in the distal half of the dorsal path (column C), were in the proximal half but not in contact with the trochanter-coxa segment boundary $(\mathrm{col}$ umn $B$ ), or were in contact with the segment boundary (column $A$ ). The Cx1 cells (when labeled) are outlined in the lower, proximal region of limbs. The degree of branching appears to increase at the midpoint of the femur (center line). This increase occurs whether or not branches have contacted the distal coxal segment boundary (the most proximal line). Calibration bar, $100 \mu \mathrm{m}$. in the most proximal region. In order to avoid the uncertainties introduced by these factors, the dorsal path was divided into four equal regions: $P_{p}, P_{d}, D_{p}$, and $D_{d}$ (Fig. 10). When the path is divided in this way, the middle two regions, $P_{d}$ and $D_{p}$, are definitely contained within the femoral segment.

Within these four regions the mean number of branches is much higher in the region just proximal to the midpoint of the femur $\left(P_{d}\right)$ than in the region just distal to the midpoint $\left(D_{p}\right)$. $P_{d}$ had a mean of $6.9(S D=3.8)$, while $D_{p}$ had a mean of 1.5 $(\mathrm{SD}=1.9)$. These differences are significant at the 0.01 level $(t$ test). Interestingly, the two most proximal regions had the same mean number of branches. This suggests that neuronal adhesion may not increase in a continuous manner along the dorsal path, but may increase with discrete steps.

\section{Increased neuronal spreading in the proximal region of the femur}

Three other types of proximal increases in degree of neuronal apposition within the femur are observed. First, at 32-33\%, growth cone branches in this region often spread and have small but distinct lamellae (Fig. 4D). Second, at about the $35 \%$ stage, pioneer axons on the dorsal path tend to show secondary spreading in the proximal region of the femur but not in the distal region (Fig. 11, C,D). The axons sometimes show an abrupt change in spreading. This correlates with the increase in branching between the distal and proximal regions of the femur (Figs. 8-10), suggesting that the general degree of neuronal apposition might change in a discrete step within the femur.

Third, a high degree of spreading of another neuron, Fel, is observed in this region. When Fe1 differentiates and begins proximal axonogenesis, its growth cone can form a broad lamellum on the epithelium (Fig. 11 $A$ ). Also, its axon tends to spread and flatten on the epithelium in the proximal femur and can be as wide as, or wider than, the Fel cell body (Fig. 11, $A$, $B$ ). In contrast, the Til growth cones and axons are much narrower than their cell bodies (Fig. $8, A, B$ ) at a comparable stage of differentiation and growth (except where they are in contact with the tibia-femur segment boundary).

\section{Discussion}

\section{Principal findings}

Figure 12 summarizes the observed spatial and temporal changes in growth cone morphology and the profile of neuronal apposition to the epithelium. Pioneer growth cones in 33-34\% embryos (upper left) cross the proximal border of the tibia, extend 


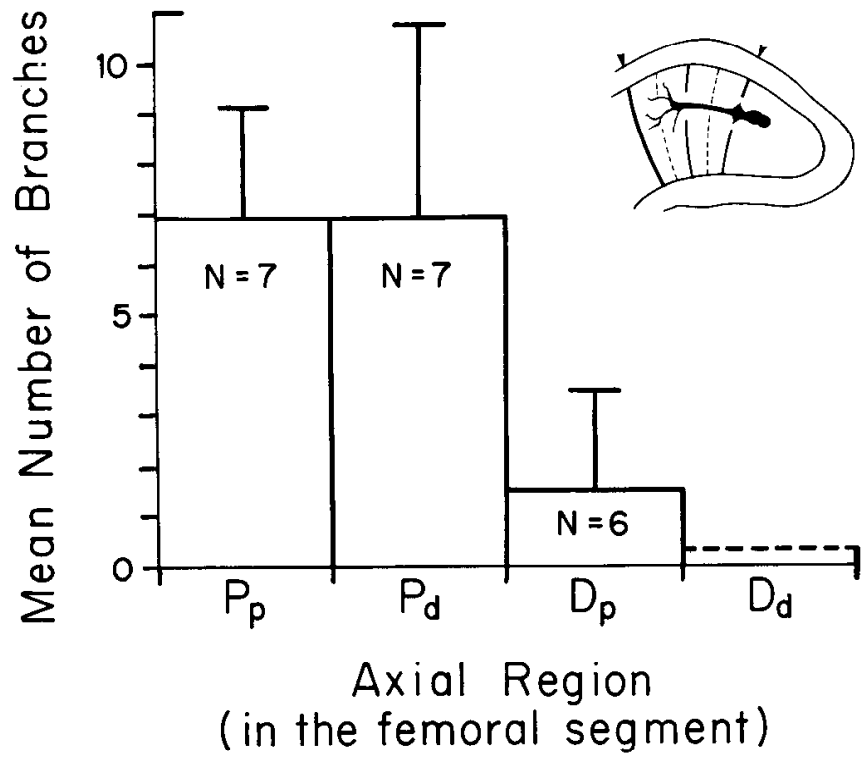

Figure 10. Mean numbers of growth cone branches in different regions of the dorsal path. The dorsal path region is divided into four equalsized regions, which are labeled (from proximal to distal) $P_{p}, P_{d}, D_{\rho}, D_{d}$. A clear difference in mean number of branches (see Methods) is evident at the midpoint of the dorsal path, which lies roughly at the midpoint of the femur. Region $D_{p}$ had a mean of 1.5 (SD 1.9), while region $P_{d}$ had a mean of 6.9 (SD 3.8). These means are different at the 0.01 level $(t$ test). However, no difference in branching is cvident between the two most proximal regions, $P_{d}$ and $P_{p}$; each had a mean of 6.9 (SD 2.2 in $P_{p} ;$ SD 3.8 in $P_{d}$ ). The distal-most region was not analyzed because none of the set of 20 legs had growth cones in this region, as determined by criteria specified in the text.

lateral lamellae or branches there, and lateral filopodia are preferentially retained (or extended) there. In PPD clutches the growth cones navigate proximally before the $\mathrm{Fcl}$ and $\mathrm{Tr} 1$ guidepost cells have differentiated. In these clutches the growth cones have a much higher degree of branching in the proximal femur than in the distal femur, and they also extend lamellae in the proximal region. When the pioneer growth cones contact the distal coxal boundary, they extend circumferential branches, discontinue proximal growth, and reorient along the boundary.

As the differentiation of leg segmentation continues during development, the previously established pioneer axons also show secondary changes in morphology (Fig. $12 ; 35-42 \% \mathrm{leg}$ ). The Til axons begin to spread and flatten on the epithelial substrate in the proximal region of the tibia but not in the distal region of the femur. This spatial contrast in morphology increases further with development. By the $42 \%$ stage, the pioneer axons have pronounced lateral branching in the proximal tibia but no branching in the distal femur.

Secondary spreading of the Ti1 axons also occurs in the proximal region of the femur after $35 \%$ but not in the more distal region. In addition, the growth cone and axon (not shown) of the Fe1 neuron exhibit a much higher degree of spreading in the proximal femur than do comparably differentiated Til growth cones in the distal femur.

The observed differences in neuronal apposition are schematized in the $\mathrm{N}$ profile below the drawings of Figure 12. In the $33-34 \%$ profile, the isolated peak at the tibia-femur boundary represents the initial spreading at this boundary that occurs before responses are observed in the adjacent regions. The peak has some width, as indicated by lamellae and clusters of filopodia. The step in the center of the femur represents the relative increase in growth cone branching observed there in PPD clutches and the abrupt secondary spreading of the Til axons at later stages. The peak at the distal coxal boundary is suggested by the lack of branch extension either proximally or distally from growth cones on the boundary. This peak also has width, as suggested by broad lamellae observed at that boundary.

In the $35-42 \%$ profile, the extension of secondary branches in the proximal tibia but not in the distal femur is represented by the abrupt drop just proximal to the tibia-femur segment boundary. The femur-trochanter segment boundary has also differentiated, but the exact profile of apposition within the trochanter has not been established by the available data.

The $E_{1}$ and $E_{2}$ profiles below the $N$ profile show two possible generalized patterns of guidance molecules on the epithelial substrate that may induce the observed changes in growth cone morphology and apposition. These will be considered below.

\section{Neuronal morphology reveals extrinsic cues}

The morphologies of pioneer growth cones correlate both spatially and temporally with the differentiation of external features in the leg. The clearest example of this is growth cone interaction with identified guidepost cells. Growth cones in contact with the Fel and $\operatorname{Tr} 1$ guidepost cells exhibit characteristic responses: They make distinct reorientations toward guidepost cells (Fig. $7 A$ ), selectively wrap them with filopodia and branchcs (Fig. $7 A$ ), and spread lamellae over them (Bentley and Caudy, 1983b). However, when the Til growth cones navigate the dorsal path before those guidepost cells have differentiated (Fig. $7 B$ ), those characteristic morphologies are not present.

Growth cone morphology and oricntation also are affected by contact with segment boundaries, the more so the more a particular boundary has differentiated. At later stages, the location of segment boundaries can be determined by staining of epithelial cells with Hoechst 33258 (Fig. $5 A$ ), as well as by the thickness and contour of the epithelium (Fig. 5B). In PPD clutches, where the tibia-femur boundary is relatively undifferentiated when the pioneer growth cones cross it, they generally show no signs of response to it (Fig. 8). However, in non-PPD clutches, where the Til growth cones navigate through a more differentiated leg, they extend lateral branches and lamellae along that boundary where they cross it (Fig. 3). Furthermore, the distance of that boundary from the Til cell bodies varies, and the lateral branches and lamellae occur at varying distances from the pioneer cell bodies (cf. Figs. $3 C$ and $11 A$ ), indicating that an internal program does not control lateral branching and spreading. Finally, contact with the even more highly differentiated distal boundary of the coxa is followed by cessation of proximal growth and circumferential reorientation of the growth cones along the boundary. Thus, neuronal morphologies reflect both the location and degree of differentiation of segment boundaries and of identified guidepost neurons.

\section{Extrinsic control of growth cone morphology in culture}

In culture, changes in concentration of external molecules-both substrate bound and freely diffusing - have been found to effect the same types of changes in growth cone morphology exhibited by pioneer growth cones in situ. These include changes in degree of branching, formation of lamellae, filopodial retention, and in general, in the degree of neuronal apposition to the substrate.

Changes in the adhesivity (i.e., the adhesion per unit area) of the substrate can cause such changes in morphology. Letourneau (1975a, b, 1979) determined the relative adhesivity of plated neurons on various substrates by measuring their resistance to lateral displacement by controlled air blasts. He then compared the morphologies of growth cones on different substrates of known relative adhesivity. Growth cones on high-adhesivity substrates spread and formed lamellar veils more often than on low-adhesion substrates (1975a), their filopodia were retained longer (1979), and their axons branched more (1975a). Growth cones that encountered an interface between regions of discretely different adhesivity reoriented to remain on the more adhesive 

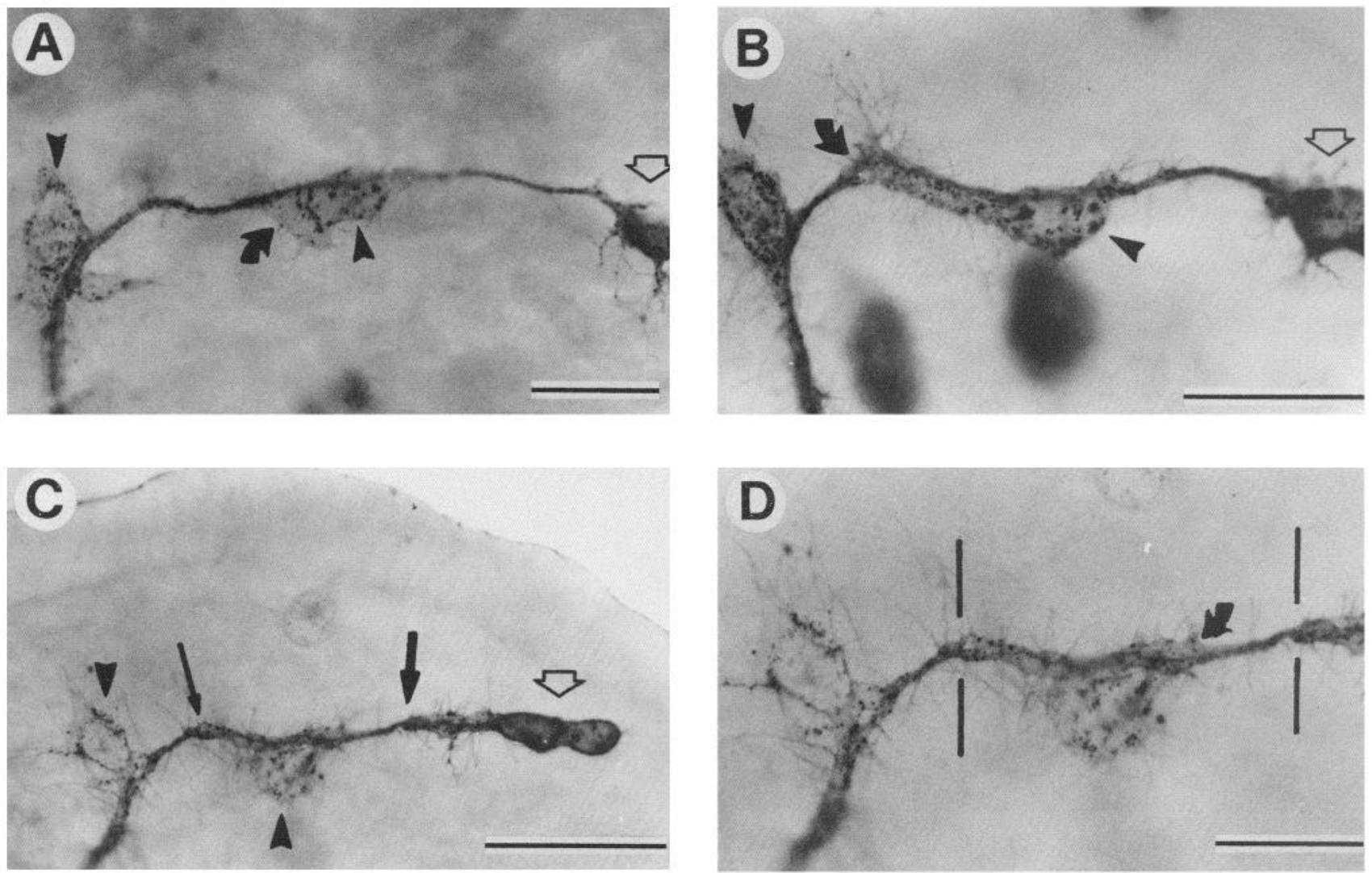

Figure 11. Morphology ef the Fel growth cones and Til pioneer axons in the proximal femur. $A$, The Fel cell body (upward carat) is located at the midpoint of the femur. At about the $36 \%$ stage, it begins axonogenesis into the proximal region of the femur. Its growth cone can take the form of a broad lamellum (curved arrow) that is even wider than the cell body. Note the lateral branches extended from the Til cell bodies (open arrow), which lie on the tibia-femur segment boundary in this leg. Open arrow, pioneer cell bodies; downward carat, $\operatorname{Tr} 1$ cell. Calibration bar, $50 \mu \mathrm{m}$. $B$, As the Fel growth cone (curved arrow) advances in the proximal region of the femur, its trailing axon sometimes spreads very widely over the epithelium. This configuration contrasts with the narrow, branchless Til growth cone at a comparable degree of differentiation in the distal region of the femur (Figs. $1 A$ and $8, A, B)$. Open arrow, pioneer cell bodies; downward carat, Tr1 cell; slanted carat, Fe1 cell body. Calibration bar, 100 $\mu \mathrm{m}$. $C$, Beginning at about the $35 \%$ stage, the Til axons also tend to spread more in the proximal region of the femur, as well as in the proximal region of the tibia (see also Fig. 5). In this leg, the axons spread in the proximal tibia, cross the tibia-femur segment boundary (larger arrow), and then become narrow in the distal femur. They spread again in the proximal femur, starting just distal to the Fel cell body (upward carat) and spreading occurs up to the femur-trochanter segment boundary (narrow arrow). They then become narrow again where they cross the femurtrochanter segment boundary into the trochanter. Open arrow, pioneer cell bodies; downward carat, Tr1 cell. Calibration bar, $100 \mu \mathrm{m} . D$, Higher magnification view of $C$. The curved arrow points to the site just distal to Fe1, where the Til axons begin to spread on the epithelium in the femur. The vertical lines indicate where the pioneer axons cross the segment boundaries marked by arrows in C. Calibration bar, $50 \mu \mathrm{m}$.

region (Hammarback et al., 1985; Letourneau, 1975b).

Increases in neuronal apposition might also be induced by external molecules that are not themselves adhesive. NGF increases adhesion (indicated by increased resistance to lateral displacement) of PC12 cell bodies (Schubert and Whitlock, 1977) and growth cones of dorsal root neurons (Gunderson and Barrett, 1980) to their substrates in culture. This increase requires metabolic energy (Schubert and Whitlock, 1977), suggesting that NGF might actively regulate local adhesion of growth cones (Connolly et al., 1985), perhaps by the local addition of adhesion molecules to the growth cone membrane. Alternatively, increased neuronal apposition might be induced by actively regulating the structure of the cytoskeleton or its attachment to the plasma membrane (Connolly et al., 1985). Local regulation of calcium influxes by NGF (Gunderson and Barrett, 1980) or other external guidance molecules may regulate the local state of the cytoskeleton (Cooper and Schliwa, 1985). As a result, increased growth cone apposition could occur without increased adhesivity of either the substrate or neuronal membrane.

We conclude that the observed changes in pioneer morphology are primarily caused by response to extrinsic cues and that neuronal morphology is a reliable indicator of such cues. How- ever, whether the extrinsic cues causing these responses are adhesive in nature or induce active response from growth cones is not unequivocally indicated by growth cone morphologies. For this reason, guidance cues will be discussed in terms of "affinity" rather than adhesion.

\section{Epithelial affinity increases proximally within segments}

\section{Extrinsic cues are highly localized on the epithelial substrate}

The observation that neuronal morphologies change radically within a very local (i.e., about $5 \mu \mathrm{m}$ ) region at segment boundaries argues against any form of global diffusion gradient generated along the length of the leg. Furthermore, the distinct asymmetries in branch extension and spreading on opposite sides of segment boundaries argue against diffusion gradients generated within each segment by source cells at the segment boundaries. There are no known membranes or other barriers at or near limb segment boundaries that could limit diffusion from a source at the segment boundary to the distal direction. Thus, highly localized extrinsic cues are indicated by the observed morphologies. These could be bound to the epithelial substrate or could be diffusible molecules released differentially 
$33-34 \%$

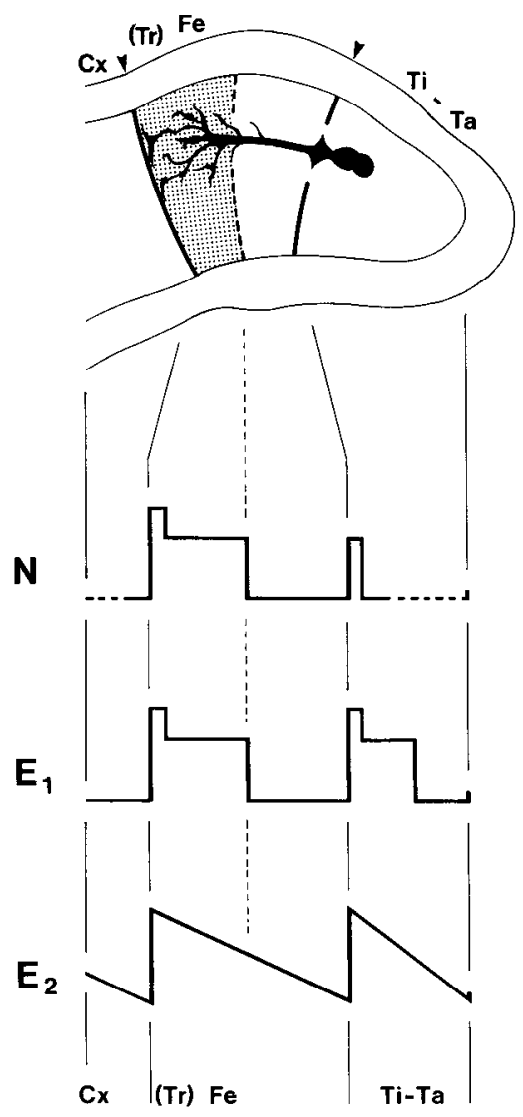

$35-42 \%$

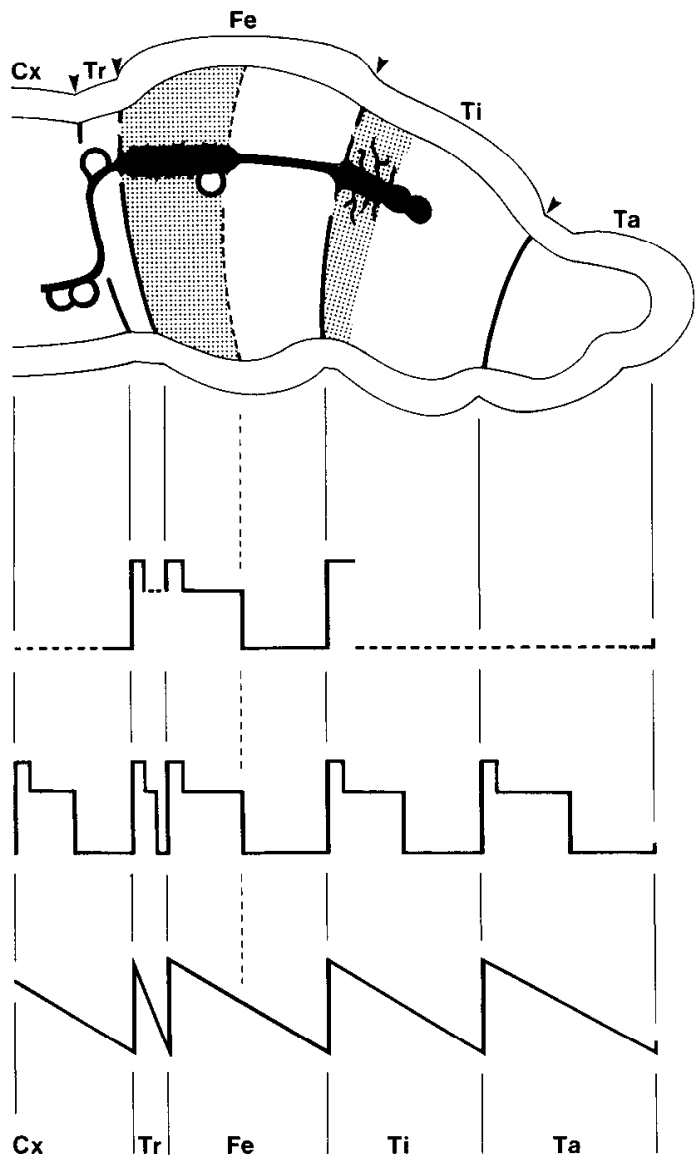

Figure 12. Diagrammatic representation of the different pioneer neuron morphologies observed at different locations and stages in the developing limb. Left, The 33-34\% stage (composite). The dorsal path runs proximally through the femur (and prospective trochanter), and in PPD clutches, the pioneer growth cones show increased branching in the proximal region of the femur. At the proximal end of the dorsal path, the pioneer axons reorient and send branches circumferentially. The pioneers also branch and spread circumferentially where they cross the tibia-femur segment boundary, as it begins to differentiate. Right, The 35-42\% stages (composite): A completed Til pathway runs proximally (along the "dorsal path") to the distal segment boundary of the coxa, where it reorients ventrally along that border and then turns to contact the pair of $\mathrm{Cx} 1$ guidepost cells in the coxa. Two other guidepost cells lie along the dorsal path: the Fel cell in the femur and the Tr1 cell at the proximal border of the trochanter. The pioneer axons have responded to further differentiation of the limb by axonal spreading and secondary branching in the proximal region of the tibia (visible at $42 \%$ ) and by axonal spreading in the proximal region of the femur (visible at $35 \%$ ). After the $33 \%$ stage, the trochanter segment begins to differentiate, and both its boundaries are evident as epithelial constrictions by $42 \%$. Branching along the femur-trochanter segment boundary can be seen after 35\%. $N$, Profile of observed differences in neuronal apposition to the epithelium, as revealed by neuronal morphology and statistical analysis of branching (sce text). $E_{1}$, One possible gencral epithelial affinity profile suggested by growth cone morphologies in different regions. A three-step increase in epithelial affinity is repeated in each segment, with a low-affinity region in the distal half of each segment, a discrete increase in epithelial affinity in the middle of the segment, and a maximum of affinity at the segment boundary. $E_{2}$, Another possible general epithelial affinity profile is a segmentally repeated, continuous gradient of proximally increasing affinity. In this case, the affinity peak at the proximal end of each segment is merely the affinity maximum at the proximal end of the gradient, not a discrete event, as in the $E_{1}$ profile. (Note: A distally increasing gradient or step might also produce the same morphologies if some active guidance molecule causes decreases in degree of neuronal apposition.) Leg segments: coxa, $C x$; trochanter, $T r$; femur, $F e$; tibia, $T i$; tarsus, $T a$.

by epithelial cells all along the segment. Either mesodermal or ectodermal cells could produce these cues. However, mesodermal cells seem unlikely to be the source: (1) in grasshopper legs, growth cones remain on the inner epithelial surface, and at this stage of limb development there is not a continuous mesodermal surface closely apposed to the epithelial substrate; and (2) in insect appendages with either reduced mesoderm (grasshopper antennae) or essentially no mesoderm (Drosophila and Manduca wing), afferent growth cones also grow proximally on the inner epithelial surface. We conclude that a local, ectodermally generated cue is involved.

\section{Neuronal apposition increases proximally within leg segments}

Characteristic neuronal morphologies-circumferentially extended branches and lamellae - are observed at segment boundaries. Similar but less pronounced morphologies - increased formation of growth cone branching and lamellae and secondary spreading of pioneer axons-are characteristic of growth cones and axons in the proximal regions of segments, but not in the distal regions. Thus, the degree of neuronal apposition to the epithelial substrate generally increases proximally within segments, with a peak at segment boundaries. Since extrinsic cues 
are apparently expressed locally by epithelial cells, the pattern of neuronal apposition suggests that epithelial affinity exhibits a similar proximal increase within segments.

Two possible epithelial affinity profiles, $E_{1}$ and $E_{2}$, are illustrated in Figure 12. The apparently abrupt onset of growth cone branching (Fig. 8) and axon spreading (Fig. 11) in the proximal region of the femur suggests that extrinsic cues may incrcasc proximally in discrete steps, rather than in continuous gradients. This pattern is illustrated in the $E_{1}$ profile. The continuous profile of $E_{2}$ is more consistent with previous studies (see below) of adhesion gradients in insect epithelium. Since the growth cones could respond in a nonlinear (stepped) fashion to a continuous epithelial gradient, our data do not distinguish between these models, and either could be present.

\section{Gradients in insect epithelium}

A proximally incrcasing gradient of surface adhesivity within the peripheral ectodermal epithelium has been proposed in the (nonsegmented) wings of moths (Nardi, 1981; Nardi and Kafatos, 1976a, b). Nardi and Kafatos (1976a, b) transplanted patches of Manduca wing epithelium from three different axial positions within the wing to one of the same threc positions in the same or another wing. They observed differences in the manner in which these patches of cells interacted with neighboring cells in the host. Initially, square patches of cells became increasingly isolated from the host epithelium (i.e., they became rounded and more highly packed) the further they were transplanted from their original axial position in the donor. Patches at ectopic locations also tended to either invaginate or evaginate, depending on the direction in which they were transplanted (Nardi, 1981). Furthermore, when two separate wing patches were juxtaposed in a neutral environment, they displayed preferential affinities for each other. Patches from the same axial position formed a single, fused epithelial vesicle, while patches from disparate positions formed two vesicles connected by a constricted region, with the degree of constriction proportional to the distance between their initial positions (Nardi and $\mathrm{Ka}$ fatos, 1976b). These various changes in shape and packing are highly consistent with a model of graded cell adhesivity within the epithelium along the wing axis.

Afferent sensory neurons are derived from the peripheral ectodermal epithelium and may therefore respond to epithelial adhesion gradients. By examining the tendency of afferent sensory nerves to either enter or avoid patches of epithelium transplanted from different axial levels in the wing, Nardi (1983; also see below) demonstrated that afferent neurons do respond to regional differences in epithelial affinity.

One explanation for the observed pattern of apposition of the Til neurons is that a proximally increasing affinity gradient occurs on the epithelial substrate in each leg segment. There is strong evidence that segmented insect epithelium can differentiate continuous gradients within each segment. Continuous segmental gradients of positional information within insect epithelium have been suggested both by graft transplantation (Locke, 1959, 1966) and by intercalary regeneration experiments (Bohn, 1970a, b, 1976; French, 1976; French et al., 1976). In both of these types of experiments, epithelial cells at every segmental position exhibited consistent, graded response to contact with cells of another segmental position. These results independently demonstrate a continuous gradient of some form of positional information, which is repeated throughout each body and limb segment.

Our data suggest a proximal increase in each limb segment of growth cone apposition regulating cues, which seem likely to be molecular in nature. These appear to be locally generated and are very probably expressed on the surface of (or locally released from) epithelial cells. Although a smoothly increasing gradient is indicated by transplant and intercalary regeneration studies of insect epithelium, our data do not distinguish between a smooth gradient (Fig. 12; $E_{2}$ profile) and a stepped increase (Fig. 12; $\mathrm{E}_{1}$ profile).

\section{Axon guidance by proximal increases in epithelial affinity}

\section{Guidance of afferent pioneer axons in insects}

Afferent pioneer neurons in insect appendages differentiate from the peripheral ectodermal epithelium, and their growth cones navigate characteristic pathways along the inner surface of the ectodermal epithelium. Much of this growth is essentially proximal in direction, although the specific characteristics of each pathway often are due to deviations from proximal growth.

Several possible guidance mechanisms depend on maintaining the physical integrity of the limb. A diffusible cue originating in the body (at least after the onset of pioneer axonogenesis) has been shown to be unnecessary by the establishment of normal sensory pathways in isolated Drosophila wing disks (Blair and Palka, 1985; Blair et al., 1985) and grasshopper antennae (Berlot and Goodman, 1984) and legs (Berlot and Goodman, 1984; Lefcort and Bentley, 1985). Channels or surface curvature, nonlocal diffusion sources, and axial electrical fields are apparently eliminated as essential guidance cues by the occurrence of normal axonogenesis after the epithelium has been cut open along the whole length of the appendage and separated into dorsal and ventral fragments (Blair and Palka, 1985; Blair et al., 1985) or opcned and pinned out flat (Lefcourt and Bentley, 1985). Normal pathfinding under these various circumstances strongly suggests guidance by internal cell polarity, which may account for the initial direction of axonogenesis (Bentley and Caudy, 1983b; Blair and Palka, 1985; Blair et al., 1985) and/or a highly localized extrinsic cue or cues.

In grasshoppers, immature neurons (guidepost cells) are the dominant cues for pioneer growth cones (Bentley and Caudy, $1983 b)$. Growth cones reorient and grow directly to the Fe 1 and Trl cells when they contact them (Fig. $7 A$ ), although those two cells are not essential for proximal pioneer growth cone navigation (Fig. 8). Thus, in grasshoppers some guidepost cells are an adequate and dominant cue when encountered but are not essential for proximal growth. In Drosophila wing, immature neurons that might serve as guideposts are also unnecesaary for normal axonogenesis (Blair and Palka, 1985; Blair et al., 1985; Schubiger and Palka, 1985). However, for at least one location in grasshopper legs, guidepost cells are essential: Selective ablation of the Cxl guidepost cells in grasshopper legs has shown them to be necessary for normal Til pioneer navigation (Bentley and Caudy, 1983a, b).

In grasshopper legs, a second prominent extrinsic cue is a limb segment boundary (Fig. 4; Bentley and Caudy, 1983b). When this boundary is sufficiently differentiated, the Til pioneer growth cones circumferentially reorient and grow along it until they make filopodial contact with the more proximally located Cx 1 guidepost cells (Fig. 4; Bentley and Caudy, 1983b). The influence of the tibia-femur segment boundary on the Til growth cones has been discussed in this paper, as has the general segmental pattern of epithelial affinity. The high affinity of segment boundaries for pioneer growth cones may be due only to their being the proximal peak in that general pattern of epithelial affinity; however, other factors may also contributc to it.

At locations other than segment boundaries, proximally increasing gradients may provide an additional extrinsic cue in insect appendages. Proximally increasing gradients that confer positional information have been proposed in insect body and leg segments (Bohn 1970a, b, 1976; French, 1976; Locke, 1959, 1966; and see above). Clever (1959) suggested that afferent sensory axons might be oriented proximally along a gradient in moth wings, and epithelial adhesion gradients have been demonstrated there (Nardi and Kafatos, 1976; see above). In moth 
wings, epithelial patch transplants have demonstrated that growing sensory axons do respond to cpithclial affinity differences, which may be adhesive in nature and which may guide afferent pioneer growth cones (Nardi, 1983). In contrast, in Drosophila wings the initial pathways taken by sensory growth cones do not appear to be oriented simply by a proximo-distal epithelial gradient (Palka et al., 1983), although such a gradient may contribute to directed growth (Blair and Palka, 1985). In grasshopper appendages, limb axis polarity cues (Bentley and Caudy, 1983b) or adhesion gradients (Berlot and Goodman, 1984) have also been proposed as a mechanism for proximal guidance, but no direct evidence for neuronal-epithelial adhesion or for axial differences in such adhesion is available (Trinkaus, 1985).

\section{Til guidance by proximal increases in epithelial affinity}

In this study, we have examined the morphologies of growth cones as they are migrating through different regions of epithelium. Pioneer growth cones exhibit a higher degree of apposition in more proximal regions of segments, with a peak at the boundary. The types of response seen-filopodial retention (or continued extension), lamellar spreading, and increased branching - are those that have been shown in culture to be caused by increased substrate adhesivity (Letourneau, 1975a, b, 1979), and similar effects are produced by nonadhesive cues such as NGF (Connolly et al., 1985). Significantly, the same factors in culture have a profound effect on the steering of growth cones: Growth cones orient to remain on substrates of higher adhesivity (Hammarback et al., 1985; Letourneau, 1975b) or higher density of surface bound NGF (Gunderson, 1985). Similarly, pioneer growth cones distinctly reorient at the distal segment boundary of the coxa, apparently to avoid contact with the low-affinity epithelium in the distal region of the coxa. At segment boundaries, pioneer growth cones exhibit prolonged retention (or extension) of filopodia and highly oriented branches, and these morphological features are likely to contribute to growth cone guidance there. Proximal increases in degree of branching and spreading also are observed as the growth cones navigate proximally through the femur. It seems likely that such pronounced morphological changes must affect growth cone guidance within segments. We conclude that in the absence of guidance by later differentiating cues, the proximal increase in epithelial affinity contributes to, and in some regions accounts for, the proximal guidance of pioneer growth cones.

\section{References}

Bate, C. M. (1976) Pioneer neurons in an insect embryo. Nature 260: $54-56$.

Bentley, D., and M. Caudy (1983a) Pioneer axons lose directed growth after selective killing of guidepost cells. Nature 304: 62-65.

Bentley, D., and M. Caudy (1983b) Navigational substrates for peripheral pioneer growth cones: limb-axis polarity cues, limb-segment boundaries, and guidepost neurons. Cold Spring Harbor Symp. Quant. Biol. 48: 573-585.

Bentley, D., and H. Keshishian (1982a) Pioneer neurons and pathways in insect appendages. Trends Neurosci. 5: 364-367.

Bentley, D., and H. Keshishian (1982b) Pathfinding by peripheral pioneer neurons in grasshoppers. Science 218: 1082-1088.

Bentley, D., H. Keshishian, M. Shankland, and A. Toroian-Raymond (1979) Quantitative staging of embryonic development of the grasshopper, Schistocerca nitens. J. Embryol. Exp. Morphol. 54: 47-74.

Berlot, J., and C. S. Goodman (1984) Guidance of peripheral pioneer neurons in the grasshopper: adhesive hierarchy of epithelial and neuronal surfaces. Science 223: 493-496.

Blair, S., and J. Palka (1985) Axon guidance in cultured wing discs and disc fragments of the Drosophila wing. Dev. Biol. 108: 411-419.

Blair, S., M. A. Murray, and J. Palka (1985) Axon guidance in cultured epithelial fragments of the Drosophila wing. Nature 315: 406-408.

Bohn, H. (1970a) Interkalare Regeneration und segmentale Gradienten bei den Extremitäten von Leucophaea-Larven (Blattaria) I. Femur und Tibia. Roux's Arch. 165: 303-341.
Bohn, H. (1970b) Interkalare Regeneration und segmentale Gradienten bei den Extremitäten von Leucophaea-Larven (Blattaria) II. Coxa und Tarsus. Dev. Biol. 23: 355-379.

Bohn, H. (1976) Regeneration of proximal tissues from a more distal amputation level in the insect leg (Blaberus cranifer, Blattaria). Dev. Biol. 53: 285-293.

Clever, U. (1959) Uber experimentelle Modifikationen des Geäders und die Beziehungen zwischen den Versorgungssystemen im Schmetterlingsflügel. Untersuchungen an Galleria mellonella. L. Roux's Arch. 151: $242-279$.

Connolly, J. L., P. J. Seeley, and L. A. Greene (1985) Regulation of growth cone morphology by nerve growth factor: A comparative study by scanning electron microscopy. J. Neurosci. Res. 13: 183-198.

Cooper, M. S., and M. Schliwa (1985) Electrical and ionic controls of tissue cell locomotion in DC electric fields. J. Neurosci. Res. 13: $223-$ 244.

French, V. (1976) Leg regeneration in the cockroach, Blattella germania. II. Regeneration from a non-congruent tibial graft/host junction. J. Embryol. Exp. Morphol. 35: 267-301.

French, V., P. Bryant, and S. Bryant (1976) Pattern regulation in epimorphic fields. Science 193: 969-981.

Goodman, C. S., K. G. Pearson, and W. J. Heitler (1979) Variability of identified neurons in grasshoppers. Comp. Biochem. Physiol. 64A: 455-462.

Gunderson, R. W. (1985) Sensory neurite growth cone guidance by substrate adsorbed nerve growth factor. J. Neurosci. Res. 13: 199212

Gunderson, R. W., and J. N. Barrett (1980) Characterisation of the turning response of dorsal root neurites toward nerve growth factor. J. Cell Biol. 87: 546-554.

Hammarback, J. A., S. L. Palm, L. T. Furcht, and P. C. Letourneau (1985) Guidance of neurite outgrowth by pathways of substratumadsorbed laminin. J. Neurosci. Res. 13: 213-220.

Ho, R. K., and C. S. Goodman (1982) Peripheral pathways are pioneered by an array of central and peripheral neurones in grasshopper embryos. Nature 297: 404-406.

Jan, L. Y., and Y. N. Jan (1982) Antibodies to horseradish peroxidase as specific neuronal markers in Drosophila and grasshopper embryos. Proc. Natl. Acad. Sci. USA 79: 2700-2704.

Keshishian, H. (1980) The origin and morphogenesis of pioneer neurons in the grasshopper metathoracic leg. Dev. Biol. 80:388-397.

Keshishian, H., and D. Bentley (1983a) Embryogenesis of peripheral nerve pathways in grasshopper legs. I. The initial nerve pathway to the CNS. Dev. Biol. 96: 89-102.

Keshishian, H., and D. Bentley (1983b) Embryogenesis of peripheral nerve pathways in grasshopper legs. II. The major nerve routes. Dev. Biol. 96: 103-115.

Keshishian, H., and D. Bentley (1983c) Embryogenesis of peripheral nerve pathways in grasshopper legs. III. Development without pioneer neurons. Dev. Biol. 96: 116-124.

Lefcort, F., and D. Bentley (1985) Neuronal guidance in dissected limb buds, in cell culture, and at ectopic locations in host limb buds. Soc. Neurosci. Abstr. 11: 334.

Letourneau, P. C. (1975a) Possible roles for cell-to-substratum adhesion in neuronal morphogenesis. Dev. Biol. 44: 77-91.

Letourneau, P. C. (1975b) Cell-to-substratum adhesion and guidance of axonal elongation. Dev. Biol. 44: 92-102.

Letourneau, P. C. (1979) Cell-substratum adhesion of neurite growth cones and its role in neurite elongation. Exp. Cell Res. 124: 127-138.

Locke, M. (1959) The cuticular pattern in an insect, Rhodnius prolixus Stal. J. Exp. Biol. 36: 459-477.

Locke, M. (1966) The cuticular pattern in an insect: the behaviour of grafts in segmented appendages. J. Insect Physiol. 12: 397-402.

Mason, C. A. (1985) Growing tips of embryonic cerebellar axons in vivo. J. Neurosci. Res. 13: 55-73.

Nardi, J. B. (1981) Epithelial invagination: adhesive properties of cells can govern position and directionality of epithelial folding. Differentiation 20: 97-103.

Nardi, J. B. (1983) Neuronal pathfinding in developing wings of the moth Manduca sexta. Dev. Biol. 95: 163-174.

Nardi, J. B., and F. Kafatos (1976a) Polarity and gradients in lepidopteran wing epidermis. I. Changes in graft polarity, form and cell density accompanying transpositions and reorientation. J. Embryol. Exp. Morphol. 36: 469-487.

Nardi, J. B., and F. Kafatos (1976b) Polarity and gradicnts in lepidopteran wing epidermis. II. The differential adhesiveness model: 
gradient of a non-diffusible cell surface parameter. J. Embryol. Exp. Morphol. 36: 489-512.

Palka, J., M. Schubiger, and R. Ellison (1983) The polarity of axon growth in the wings of Drosophila melanogaster. Dev. Biol. 98: 481492.

Schubert, D., and C. Whitlock (1977) Alteration of cellular adhesion by nerve growth factor. Proc. Natl. Acad. Sci. USA 74: 4055-4058.

Schubiger, M., and J. Palka (1985) Genetic suppression of putative guidepost cells: effect on establishment of nerve pathways in Drosophila wings. Dev. Biol. 108: 399-410.
Steeves, J. D., and K. G. Pearson (1983) Variability in the structure of an identified interneurone in isogeneic clones of locusts. J. Exp. Biol. 103: 47-54.

Taghert, P. H., M. J. Bastiani, R. K. Ho, and C. S. Goodman (1982) Guidance of pioneer growth cones: filopodial contacts and coupling revealed with an antibody to lucifer yellow. Dev. Biol. 94: 391-399. Trinkaus, J. P. (1985) Further thoughts on directional cell movement during morphogenesis. J. Neurosci. Res. 13: 1-19. 